\title{
Equality of Linear and Symplectic Orbits
}

\author{
Pratyusha Chattopadhyay and Ravi A. Rao \\ Stat-Math Unit, Indian Statistical Institute, 203 B.T. Road, Kolkata 700 108, India \& \\ Tata Institute of Fundamental Research, Dr. Homi Bhabha Road, Mumbai 400 005, India
}

2000 Mathematics Subject Classification: 13C10, 13H05, 15A63, 19A13, 19B10, $19 B 14$

Key words: unimodular rows, transvections, elementary symplectic group

\begin{abstract}
It is shown that the set of orbits of the action of the elementary symplectic transvection group on all unimodular elements of a symplectic module over a commutative ring in which 2 is invertible is identical with the set of orbits of the action of the corresponding elementary transvection group. This result is used to get improved injective stability estimates for $K_{1}$ of the symplectic transvection group over a non-singular affine algebras.
\end{abstract}

\section{Introduction}

In this paper we discuss two related questions about (linear and symplectic) elementary transvections of a projective $R$-module (resp. symplectic $R$-module) of type $R \oplus P($ resp. $(\mathbb{H}(R) \oplus(P,\langle\rangle))$,$) .$

The first one is about comparing the groups generated by the two different types of elementary transvections; and showing that they are the same. (This fact seems to have escaped notice earlier; and experts have told us that it is interesting, and opens up the study done on these transvections.)

The second one is to show that the linear and symplectic elementary transvection orbits of a unimodular element in a symplectic module coincide. (This generalizes the result in [7] where it was shown in the free case.)

We now describe the two problems a bit more in detail.

$\mathrm{H}$. Bass introduced two types of linear transvections of a projective module $R \oplus P$ in [3. He also introduced two types of symplectic transvecitons of a symplectic module $\mathbb{H}(R) \oplus(P,\langle\rangle$,$) . (These are recalled in \S 4$ and $\S 5)$.

Since elementary automorphisms are homotopic to the identity, we are able to invoke Quillen-Suslin theory (see [14, [17]) to show that

- the groups generated by the two types of elementary linear transvections are the same as the elementary linear group in the free case (see Lemma 4.5).

- the group generated by the two types of elementary symplectic transvections w.r.t. the standard alternating form are the same as the elementary symplectic group in the free case (see Lemma [5.14). 
The above generalizes the special case of these results in (6 , Theorem 2).

The title of this paper alludes to the comparison of the elementary linear and elementary symplectic orbits of a unimodular element $(a, b, p)$ in a symplectic module $(\mathbb{H}(R) \oplus P)$. In case $P$ is free of rank $\geq 4$, it is established in ([7], Theorem 4.2, Theorem 5.6) that these two orbits coincide. In the Appendix the missing case when $P$ is free of rank 2 is proved by a similar, but slightly more involved argument. (This means one has to essentially prove Lemma 2.9 and Lemma 3.1 in [7.)

Since elementary automorphisms are homotopic to the identity, we show how the Quillen-Suslin machinery enables one to extend the results of [7] to show that the two orbits are equal in the general case when $P$ is a finitely generated projective module. (The transition is by no means automatic!)

\section{Preliminaries}

A row $v=\left(v_{1}, \ldots, v_{n}\right) \in R^{n}$ is said to be unimodular if there are elements $w_{1}, \ldots, w_{n}$ in $R$ such that $v_{1} w_{1}+\cdots+v_{n} w_{n}=1 . \operatorname{Um}_{n}(R)$ will denote the set of all unimodular rows $v \in R^{n}$. Let $I$ be an ideal in $R$. We denote by $\operatorname{Um}_{n}(R, I)$ the set of all unimodular rows of length $n$ which are congruent to $e_{1}=(1,0, \ldots, 0)$ modulo $I$. (If $I=R$, then $\operatorname{Um}_{n}(R, I)$ is $\left.\operatorname{Um}_{n}(R)\right)$.

Definition 2.1. Let $P$ be a finitely generated projective $R$-module. An element $p \in P$ is said to be unimodular if there exists a $R$-linear map $\phi: P \rightarrow R$ such that $\phi(p)=1$. The collection of unimodular elements of $P$ is denoted by $\operatorname{Um}(P)$.

Let $P$ be of the form $R \oplus Q$ and have an element of the form $(1,0)$ which correspond to the unimodular element. An element $(a, q) \in P$ is said to be relative unimodular w.r.t. an ideal $I$ of $R$ if $(a, q)$ is unimodular and $(a, q)$ is congruent to $(1,0)$ modulo $I P$. The collection of all relative unimodular elements w.r.t. an ideal $I$ is denoted by $\mathrm{Um}(P, I P)$.

Let us recall that if $M$ is a finitely presented $R$-module and $S$ is a multiplicative set of $R$, then $S^{-1} \operatorname{Hom}_{R}(M, R) \cong \operatorname{Hom}_{R_{S}}\left(M_{S}, R_{S}\right)$. Also recall that if $f=\left(f_{1}, \ldots, f_{n}\right) \in$ $R^{n}:=M$, then $\Theta_{M}(f)=\{\phi(f): \phi \in \operatorname{Hom}(M, R)\}=\sum_{i=1}^{n} R f_{i}$. Therefore, if $P$ is a finitely generated projective $R$-module of rank $n, \mathfrak{m}$ is a maximal ideal of $R$ and $v \in$ $\operatorname{Um}(P)$, then $v_{\mathfrak{m}} \in \operatorname{Um}_{n}\left(R_{\mathfrak{m}}\right)$. Similarly if $v \in \operatorname{Um}(P, I P)$ then $v_{\mathfrak{m}} \in \operatorname{Um}_{n}\left(R_{\mathfrak{m}}, I_{\mathfrak{m}}\right)$.

The group $\mathrm{GL}_{n}(R)$ of invertible matrices acts on $R^{n}$ in a natural way: $v \longrightarrow v \sigma$, if $v \in R^{n}, \sigma \in \mathrm{GL}_{n}(R)$. This map preserves $\mathrm{Um}_{n}(R)$, so $\mathrm{GL}_{n}(R)$ acts on $\mathrm{Um}_{n}(R)$. Note that any subgroup $\mathrm{G}$ of $\mathrm{GL}_{n}(R)$ also acts on $\operatorname{Um}_{n}(R)$. Let $v, w \in \operatorname{Um}_{n}(R)$, we denote $v \sim_{\mathrm{G}} w$ or $v \in w \mathrm{G}$ if there is a $g \in \mathrm{G}$ such that $v=w g$.

Let $\mathrm{E}_{n}(R)$ denote the subgroup of $\mathrm{SL}_{n}(R)$ consisting of all elementary matrices, i.e. those matrices which are a finite product of the elementary generators $\mathrm{E}_{i j}(\lambda)=$ $I_{n}+e_{i j}(\lambda), 1 \leq i \neq j \leq n, \lambda \in R$, where $e_{i j}(\lambda) \in \mathrm{M}_{n}(R)$ has an entry $\lambda$ in its $(i, j)$-th position and zero elsewhere.

In the sequel, if $\alpha$ denotes an $m \times n$ matrix, then we let $\alpha^{t}$ denote its transpose matrix. This is of course an $n \times m$ matrix. However, we will mostly be working with square matrices, or rows and columns. 
Definition 2.2. The Relative Groups $\mathbf{E}_{n}(I), \mathbf{E}_{n}(R, I)$ : Let $I$ be an ideal of $R$. The relative elementary group $\mathrm{E}_{n}(I)$ is the subgroup of $\mathrm{E}_{n}(R)$ generated as a group by the elements $\mathrm{E}_{i j}(x), x \in I, 1 \leq i \neq j \leq n$.

The relative elementary group $\mathrm{E}_{n}(R, I)$ is the normal closure of $\mathrm{E}_{n}(I)$ in $\mathrm{E}_{n}(R)$.

(Equivalently, $\mathrm{E}_{n}(R, I)$ is generated as a group by $\mathrm{E}_{i j}(a) \mathrm{E}_{j i}(x) E_{i j}(-a)$, with $a \in$ $R, x \in I, i \neq j$, provided $n \geq 3$ (see [16], Section 2)).

Definition 2.3. $\mathbf{E}_{n}^{1}(R, I)$ is the subgroup of $\mathrm{E}_{n}(R)$ generated by the elements of the form $E_{1 i}(a)$ and $E_{j 1}(x)$, where $a \in R, x \in I$, and $2 \leq i, j \leq n$.

Remark 2.4. It is easy to check that if $v \in \operatorname{Um}_{n}(R, I)$, where $(R, \mathfrak{m})$ is a local ring and $I$ be an ideal of $R$, then $v=e_{1} \beta$, for some $\beta \in \mathrm{E}_{n}(R, I)$.

Definition 2.5. Symplectic Group $\mathbf{S p}_{2 n}(R)$ : The symplectic group $\operatorname{Sp}_{2 n}(R)=$ $\left\{\alpha \in \mathrm{GL}_{2 n}(R) \mid \alpha^{t} \psi_{n} \alpha=\psi_{n}\right\}$, where $\psi_{n}=\sum_{i=1}^{n} e_{2 i-1,2 i}-\sum_{i=1}^{n} e_{2 i, 2 i-1}$, the standard symplectic form.

Let $\sigma$ denote the permutation of the natural numbers given by $\sigma(2 i)=2 i-1$ and $\sigma(2 i-1)=2 i$.

Definition 2.6. Elementary Symplectic Group $\operatorname{ESp}_{2 n}(R)$ : We define for $z \in R$, $1 \leq i \neq j \leq 2 n$,

$$
s e_{i j}(z)= \begin{cases}1_{2 n}+z e_{i j} & \text { if } i=\sigma(j), \\ 1_{2 n}+z e_{i j}-(-1)^{i+j} z e_{\sigma(j) \sigma(i)} & \text { if } i \neq \sigma(j) .\end{cases}
$$

(It is easy to check that all these elements belong to $\operatorname{Sp}_{2 n}(R)$ ).

We call them elementary symplectic matrices over $R$ and the subgroup of $\operatorname{Sp}_{2 n}(R)$ generated by them is called the elementary symplectic group $\operatorname{ESp}_{2 n}(R)$.

Definition 2.7. The Relative Group $\mathbf{E S p}_{2 n}(I), \mathbf{E S p}_{2 n}(R, I)$ : Let $I$ be an ideal of $R$. The relative elementary group $\mathrm{ESp}_{2 n}(I)$ is the subgroup of $\mathrm{ESp}_{2 n}(R)$ generated as a group by the elements $\operatorname{se}_{i j}(x), x \in I$ and $1 \leq i \neq j \leq 2 n$.

The relative elementary group $\mathrm{ESp}_{2 n}(R, I)$ is the normal closure of $\mathrm{ESp}_{2 n}(I)$ in $\mathrm{ESp}_{2 n}(R)$.

Definition 2.8. The group $\operatorname{ESp}_{2 n}^{1}(R, I)$ is the subgroup of $\operatorname{ESp}_{2 n}(R)$ generated by the elements of the form $s_{1 i}(a)$ and $s_{j 1}(x)$, where $a \in R, x \in I$ and $2 \leq i, j \leq 2 n$.

Notation 2.9. We fix some notations. $\mathrm{G}(n, R)$ will denote either the linear group $\mathrm{GL}_{n}(R)$, or the symplectic group $\operatorname{Sp}_{2 m}(R)$, for $n=2 m$. Also, $\mathrm{E}(n, R)$ will denote either of the elementary subgroups $\mathrm{E}_{n}(R)$ or $\mathrm{ESp}_{2 m}(R)$. The standard elementary generators of $\mathrm{E}(n, R)$ are denoted by $g e_{i j}(a), a \in R$. Let $I$ be an ideal in $R$. Let $\mathrm{G}(n, R, I)$ denote the relative linear groups $\mathrm{GL}_{n}(R, I)$, or the relative symplectic group $\operatorname{Sp}_{2 m}(R, I)$. Note that $\mathrm{E}(n, I)$ is a subgroup of $\mathrm{E}(n, R)$ generated as a group by the elements $g e_{i j}(x)$, where $x \in I$, and $1 \leq i \neq j \leq n$. Here, $\mathrm{E}(n, R, I)$ denotes the corresponding relative elementary subgroups $\mathrm{E}_{n}(R, I), \operatorname{ESp}_{2 m}(R, I)$, respectively. 
These are the normal closures of the subgroups $\mathrm{E}(n, I)$ in $\mathrm{E}(n, R)$, which are also known to be generated by the elements $g e_{i j}(a) g e_{j i}(x) g e_{i j}(-a), a \in R, x \in I$, and $1 \leq i \neq j \leq n$ (see [20, Lemma 8). Also, $\mathrm{E}^{1}(n, R, I)$ is a subgroup of $\mathrm{E}(n, R)$, generated by the elements $g e_{1 i}(a)$, where $a \in R$ and $g e_{j 1}(x)$, where $x \in I, 2 \leq i, j \leq n$ in the linear and symplectic case.

Lemma 2.10. Let $n \geq 3$ in the linear case and $n \geq 4$ in the symplectic case. Let $R$ be a commutative ring with $R=2 R$, and let $I$ be an ideal of $R$. Then the following sequence is exact

$$
1 \longrightarrow \mathrm{E}(n, R, I) \longrightarrow \mathrm{E}^{1}(n, R, I) \longrightarrow \mathrm{E}^{1}(n, R / I, 0) \longrightarrow 1 .
$$

Thus $\mathrm{E}(n, R, I)$ equals $\mathrm{E}^{1}(n, R, I) \cap \mathrm{G}(n, R, I)$.

Proof: For the proof in the linear case reader can see (10, Lemma 2.2). The proof in the symplectic case is similar when $n \geq 6$. The proof when $n=4$ is discussed in the Appendix.

\section{Local Global Principle for Relative Elementary Group}

In this section we reprove the Local-Global Principle for an extended ideal, done in [1, by a different approach. The idea in 1 is to use the special forms (as described by Suslin in [17] for the linear group, Kopeiko in [11] for the symplectic group, and SuslinKopeiko in [18 for the orthogonal groups). The idea here is to use a slightly larger group $\mathrm{E}^{1}(n, R, I)$ than the relative groups $\mathrm{E}(n, R, I)$. This group was introduced by $\mathrm{W}$. van der Kallen in [10]. Note that the absolute case (when $I=R$ ) was first done in 2] again by a different approach. We proceed to describe this argument now.

Lemma 3.1. Let $R$ be a commutative ring with $R=2 R$, and let $I$ be an ideal of $R$. Let $n \geq 3$ in the linear case and $n \geq 4$ in the symplectic case. Let $\varepsilon=\varepsilon_{1} \ldots \varepsilon_{r}$ be an element of $\mathrm{E}^{1}(n, R, I)$, where each $\varepsilon_{k}$ is an elementary generator. If $g e_{i j}(X f(X))$ is an elementary generator of $\mathrm{E}^{1}(n, R[X], I[X])$, then

$$
\varepsilon g e_{i j}\left(Y^{4^{r}} X f\left(Y^{4^{r}} X\right)\right) \varepsilon^{-1}=\prod_{t=1}^{s} g e_{i_{t} j_{t}}\left(Y h_{t}(X, Y)\right)
$$

where either $i_{t}=1$ or $j_{t}=1$ and $h_{t}(X, Y) \in R[X, Y]$, when $i_{t}=1 ; h_{t}(X, Y) \in I[X, Y]$ when $j_{t}=1$.

Proof: Proof in the linear case is straight forward. We establish the result here in the symplectic case when $n \geq 6$. In the Appendix the case when $n=4$ is also covered.

We prove the result using induction on $r$, where $\varepsilon$ is product of $r$ many elementary generators. Let $r=1$ and $\varepsilon=s e_{p q}(a)$. Note that $a \in R$ when $p=1$, and $a \in I$ when $q=1$. Also, note that we use $*$ to represent elements of the ideal $I$. Given that $s e_{i j}(X f(X))$ is an elementary generator of $\operatorname{ESp}_{2 n}^{1}(n, R[X], I[X])$. First we assume $i=1$ and $j \neq 2$, hence $f(X) \in R[X]$. 
Case (1): Let $(p, q)=(1, j)$. In this case

$$
s e_{1 j}(a) s e_{1 j}\left(Y^{4} X f\left(Y^{4} X\right)\right) s e_{1 j}(-a)=s e_{1 j}\left(Y^{4} X f\left(Y^{4} X\right)\right) \text {. }
$$

Case (2): Let $(p, q)=(1, \sigma(j))$. In this case

$$
\begin{aligned}
& s e_{1 \sigma(j)}(a) s e_{1 j}\left(Y^{4} X f\left(Y^{4} X\right)\right) s e_{1 \sigma(j)}(-a) \\
= & s e_{12}\left(-a Y^{4} X f\left(Y^{4} X\right)\right) s e_{1 j}\left(Y^{4} X f\left(Y^{4} X\right)\right) .
\end{aligned}
$$

Case (3): Let $(p, q)=(1, k), k \neq j, \sigma(j)$. In this case

$$
s e_{1 k}(a) s e_{1 j}\left(Y^{4} X f\left(Y^{4} X\right)\right) s e_{1 k}(-a)=s e_{1 j}\left(Y^{4} X f\left(Y^{4} X\right)\right) \text {. }
$$

Case (4): Let $(p, q)=(2,1)$. In this case

$$
\begin{aligned}
& s e_{21}(a) s e_{1 j}\left(Y^{4} X f\left(Y^{4} X\right)\right) s e_{21}(-a) \\
= & s e_{2 j}\left(* Y^{4} X f\left(Y^{4} X\right)\right) s e_{\sigma(j) j}\left(* Y^{4} X f\left(Y^{4} X\right)\right) s e_{1 j}\left(Y^{4} X f\left(Y^{4} X\right)\right) \\
= & s e_{\sigma(j) 1}\left(* Y^{4} X f\left(Y^{4} X\right)\right)\left[s e_{\sigma(j) 1}\left(* Y^{2}\right), s e_{1 j}\left(Y^{2} X f\left(Y^{4} X\right)\right)\right] \\
& s e_{1 j}\left(Y^{4} X f\left(Y^{4} X\right)\right) .
\end{aligned}
$$

Case (5): Let $(p, q)=(k, 1), k \neq 2, j, \sigma(j)$. In this case

$$
\begin{aligned}
& s e_{k 1}(a) s e_{1 j}\left(Y^{4} X f\left(Y^{4} X\right)\right) s e_{k 1}(-a) \\
= & s e_{k j}\left(* Y^{4} X f\left(Y^{4} X\right)\right) s e_{1 j}\left(Y^{4} X f\left(Y^{4} X\right)\right) \\
= & {\left[s e_{k 1}\left(* Y^{2}\right), s e_{1 j}\left(Y^{2} X f\left(Y^{4} X\right)\right)\right] s e_{1 j}\left(Y^{4} X f\left(Y^{4} X\right)\right) . }
\end{aligned}
$$

Case (6): Let $(p, q)=(\sigma(j), 1)$. In this case

$$
\begin{aligned}
& s e_{\sigma(j) 1}(a) s e_{1 j}\left(Y^{4} X f\left(Y^{4} X\right)\right) s e_{\sigma(j) 1}(-a) \\
= & s e_{\sigma(j) j}\left(* Y^{4} X f\left(Y^{4} X\right)\right) s e_{1 j}\left(Y^{4} X f\left(Y^{4} X\right)\right) \\
= & {\left[s e_{\sigma(j) 1}\left(* Y^{2}\right), s e_{1 j}\left(Y^{2} X f\left(Y^{4} X\right)\right)\right] s e_{1 j}\left(Y^{4} X f\left(Y^{4} X\right)\right) . }
\end{aligned}
$$

Case (7): Let $(p, q)=(j, 1)$. Let us choose an integer $d \neq 1,2, j, \sigma(j)$. In this case

$$
\begin{aligned}
& s e_{j 1}(a) s e_{1 j}\left(Y^{4} X f\left(Y^{4} X\right)\right) s e_{j 1}(-a) \\
= & s e_{j 1}(a)\left[s e_{1 d}\left(Y^{2} X f\left(Y^{4} X\right)\right), s e_{d j}\left(Y^{2}\right)\right] s e_{j 1}(-a) \\
= & {\left[s e_{j d}\left(* Y^{2} X f\left(Y^{4} X\right)\right) s e_{1 d}\left(Y^{2} X f\left(Y^{4} X\right)\right), s e_{d 1}\left(* Y^{2}\right) s e_{d j}\left(Y^{2}\right)\right] } \\
= & s e_{j d}\left(* Y^{2} X f\left(Y^{4} X\right)\right) s e_{1 d}\left(Y^{2} X f\left(Y^{4} X\right)\right) s e_{d 1}\left(* Y^{2}\right) s e_{d j}\left(Y^{2}\right) \\
& s e_{1 d}\left(-Y^{2} X f\left(Y^{4} X\right)\right) s e_{j d}\left(-* Y^{2} X f\left(Y^{4} X\right)\right) s e_{d j}\left(-Y^{2}\right) s e_{d 1}\left(-* Y^{2}\right) \\
= & s e_{j d}\left(* Y^{2} X f\left(Y^{4} X\right)\right) s e_{1 d}\left(Y^{2} X f\left(Y^{4} X\right)\right) s e_{d 1}\left(* Y^{2}\right) s e_{d j}\left(Y^{2}\right) \\
& s e_{1 d}\left(-Y^{2} X f\left(Y^{4} X\right)\right)\left[s e_{j 1}(-* Y), s e_{1 d}\left(Y X f\left(Y^{4} X\right)\right)\right] s e_{d j}\left(-Y^{2}\right) s e_{d 1}\left(-* Y^{2}\right) \\
= & s e_{j d}\left(* Y^{2} X f\left(Y^{4} X\right)\right) s e_{1 d}\left(Y^{2} X f\left(Y^{4} X\right)\right) s e_{d 1}\left(* Y^{2}\right) s e_{d j}\left(Y^{2}\right) \\
& s e_{1 d}\left(-Y^{2} X f\left(Y^{4} X\right)\right) s e_{d j}\left(-Y^{2}\right) s e_{d j}\left(Y^{2}\right) \\
& {\left[s e_{j 1}(-* Y), s e_{1 d}\left(Y X f\left(Y^{4} X\right)\right)\right] s e_{d j}\left(-Y^{2}\right) s e_{d 1}\left(-* Y^{2}\right) } \\
= & {\left[s e_{j 1}(* Y), s e_{1 d}\left(Y X f\left(Y^{4} X\right)\right)\right] s e_{1 d}\left(Y^{2} X f\left(Y^{4} X\right)\right) s e_{d 1}\left(* Y^{2}\right) } \\
& s e_{1 d}\left(-Y^{2} X f\left(Y^{4} X\right)\right) s e_{1 j}\left(Y^{4} X f\left(Y^{4} X\right)\right)\left[s e_{d 1}\left(-* Y^{3}\right) s e_{j 1}(-* Y),\right. \\
& \left.s e_{1 j}\left(-Y^{3} X f\left(Y^{4} X\right)\right) s e_{1 d}\left(Y X f\left(Y^{4} X\right)\right)\right] s e_{d 1}\left(* Y^{2}\right) .
\end{aligned}
$$


Now we consider the case when $(i, j)=(1,2)$.

Case (8): Let $(p, q)=(1,2)$. In this case

$$
s e_{12}(a) s e_{12}\left(Y^{4} X f\left(Y^{4} X\right)\right) s e_{12}(-a)=s e_{12}\left(Y^{4} X f\left(Y^{4} X\right) .\right.
$$

Case (9): Let $(p, q)=(1, k), k \neq 2$. In this case

$$
s e_{1 k}(a) s e_{12}\left(Y^{4} X f\left(Y^{4} X\right)\right) s e_{1 k}(-a)=s e_{12}\left(Y^{4} X f\left(Y^{4} X\right) .\right.
$$

Case (10): Let $(p, q)=(k, 1), k \neq 2$. In this case

$$
\begin{aligned}
& s e_{k 1}(a) s e_{12}\left(Y^{4} X f\left(Y^{4} X\right)\right) s e_{k 1}(-a) \\
= & s e_{1 \sigma(k)}\left(* Y^{4} X f\left(Y^{4} X\right)\right) s e_{k \sigma(k)}\left(* Y^{4} X f\left(Y^{4} X\right)\right) s e_{12}\left(Y^{4} X f\left(Y^{4} X\right)\right) \\
= & s e_{1 \sigma(k)}\left(* Y^{4} X f\left(Y^{4} X\right)\right)\left[s e_{k 1}\left(* Y^{2}\right), s e_{1 \sigma(k)}\left(Y^{2} X f\left(Y^{4} X\right)\right)\right] \\
& s e_{12}\left(Y^{4} X f\left(Y^{4} X\right)\right) .
\end{aligned}
$$

Case (11): Let $(p, q)=(2,1)$. Let us choose an integer $d \neq 1,2$. In this case

$$
\begin{aligned}
& s e_{21}(a) s e_{12}\left(Y^{4} X f\left(Y^{4} X\right)\right) s e_{21}(-a) \\
= & s e_{21}(a)\left[s e_{1 d}\left(Y^{2} / 2\right), s e_{d 2}\left(Y^{2} X f\left(Y^{4} X\right)\right)\right] s e_{21}(-a) \\
= & {\left[s e_{2 d}\left(* Y^{2}\right) s e_{\sigma(d) d}\left(* Y^{4}\right) s e_{1 d}\left(Y^{2} / 2\right), s e_{2 \sigma(d)}\left(* Y^{2} X f\left(Y^{4} X\right)\right)\right.} \\
& \left.s e_{d \sigma(d)}\left(* Y^{4} X^{2} f^{2}\left(Y^{4} X\right)\right) s e_{d 2}\left(Y^{2} X f\left(Y^{4} X\right)\right)\right] \\
= & {\left[s e_{\sigma(d) 1}\left(* Y^{2}\right)\left[s e_{\sigma(d) 1}\left(* Y^{2}\right), s e_{1 d}\left(Y^{2}\right)\right] s e_{1 d}\left(Y^{2} / 2\right), s e_{d 1}\left(* Y^{2} X f\left(Y^{4} X\right)\right)\right.} \\
& {\left.\left[s e_{d 1}\left(* Y^{2}\right), s e_{1 \sigma(d)}\left(Y^{2} X^{2} f^{2}\left(Y^{4} X\right)\right)\right] s e_{1 \sigma(d)}\left(Y^{2} X f\left(Y^{4} X\right)\right)\right] }
\end{aligned}
$$

Hence the result is true when $i=1$ and $\varepsilon$ is an elementary generator. Carrying out similar calculation one can show the result is true when $j=1$ and $\varepsilon$ is an elementary generator. Let us assume that the result is true when $\varepsilon$ is product of $r-1$ many elementary generators, i.e, $\varepsilon_{2} \ldots \varepsilon_{r} s e_{i j}\left(Y^{4^{r-1}} X f\left(Y^{4^{r-1}}(X)\right) \varepsilon_{r}^{-1} \ldots \varepsilon_{2}^{-1}=\right.$ $\prod_{t=1}^{k} s e_{p_{t} q_{t}}\left(Y g_{t}(X, Y)\right)$, where either $p_{t}=1$ or $q_{t}=1$. Note that $g_{t}(X, Y) \in R[X, Y]$ when $p_{t}=1$ and $g_{t}(X, Y) \in I[X, Y]$ when $q_{t}=1$.

We now establish the result when $\varepsilon$ is product of $r$ many elementary generators. We have

$$
\begin{aligned}
& \varepsilon s e_{i j}\left(Y^{4^{r}} X f\left(Y^{4^{r}} X\right)\right) \varepsilon^{-1} \\
= & \varepsilon_{1} \varepsilon_{2} \ldots \varepsilon_{r} s e_{i j}\left(Y^{4^{r}} X f\left(Y^{4^{r}} X\right)\right) \varepsilon_{r}^{-1} \ldots \varepsilon_{2}^{-1} \varepsilon_{1}^{-1} \\
= & \varepsilon_{1}\left(\prod_{t=1}^{k} s e_{p_{t} q_{t}}\left(Y^{4} g_{t}^{\prime}(X, Y)\right)\right) \varepsilon_{1}^{-1} \\
= & \prod_{t=1}^{k} \varepsilon_{1} s e_{p_{t} q_{t}}\left(Y^{4} g_{t}^{\prime}(X, Y)\right) \varepsilon_{1}^{-1} \\
= & \prod_{t=1}^{s} s e_{i_{t} j_{t}}\left(Y h_{t}(X, Y)\right) .
\end{aligned}
$$


To get the last equality one needs to repeat the calculation which was done for a single elementary generator. Note that at the last line either $i_{t}=1$ or $j_{t}=1$. Also, note that $h_{t}(X, Y) \in R[X, Y]$, when $i_{t}=1$ and $h_{t}(X, Y) \in I[X, Y]$, when $j_{t}=1$

Notation 3.2. Let $M$ be a finitely presented $R$-module and $a$ be a non-nilpotent element of $R$. Let $R_{a}$ denote the ring $R$ localized at the multiplicative set $\left\{a^{i}: i \geq 0\right\}$ and $M_{a}$ denote the $R_{a}$-module $M$ localized at $\left\{a^{i}: i \geq 0\right\}$. Let $\alpha(X)$ be an element of $\operatorname{End}(M[X])$. The localization map $i: M \rightarrow M_{a}$ induces a map $i^{*}: \operatorname{End}(M[X]) \rightarrow$ $\operatorname{End}\left(M[X]_{a}\right)=\operatorname{End}\left(M_{a}[X]\right)$. We shall denote $i^{*}(\alpha(X))$ by $\alpha(X)_{a}$ in the sequel.

Lemma 3.3. Let $M$ be a finitely presented $R$-module and $I$ be an ideal of $R$. Let $\alpha(X), \beta(X) \in \operatorname{End}(M[X], I M[X])=\operatorname{ker}(\operatorname{End}(M[X]) \longrightarrow \operatorname{End}(M[X] / I M[X]))$, with $\alpha(0)=\beta(0)$. Let a be a non-nilpotent element in $R$ such that $\alpha(X)_{a}=\beta(X)_{a}$ in $\operatorname{End}\left(M_{a}[X], I M_{a}[X]\right)$. Then $\alpha\left(a^{N} X\right)=\beta\left(a^{N} X\right)$ in $\operatorname{End}(M[X], I M[X])$, for $N \gg 0$.

Proof: Using $\alpha(0)-\beta(0)=0$, we get $\alpha(X)-\beta(X)=X \gamma(X)$, for some $\gamma(X)$ in $\operatorname{End}(M[X], I M[X])$. Also $\alpha(X)_{a}-\beta(X)_{a}=0$ in $\operatorname{End}\left(M_{a}[X], I M_{a}[X]\right)$, i.e, $(\alpha(X)-$ $\beta(X))_{a}=0$, i.e, $(X \gamma(X))_{a}=0$. Hence $a^{N}(X \gamma(X))=0$, in $\operatorname{End}(M[X], I M[X])$, for some $N \gg 0$. Therefore $\alpha\left(a^{N} X\right)-\beta\left(a^{N} X\right)=a^{N} X \gamma\left(a^{N} X\right)=0$, in $\operatorname{End}(M[X], I M[X])$, for $N \gg 0$.

Lemma 3.4. Let $R$ be a commutative ring with $R=2 R$, and let $I$ be an ideal of $R$. Let $n \geq 3$ in the linear case and $n \geq 4$ in the symplectic case. Let a be a non-nilpotent element in $R$ and $\alpha(X)$ be in $\mathrm{E}^{1}\left(n, R_{a}[X], I_{a}[X]\right)$, with $\alpha(0)=I d$. Then there exists $\alpha^{*}(X) \in \mathrm{E}^{1}(n, R[X], I[X])$, with $\alpha^{*}(0)=I d$., such that $\alpha^{*}(X)$ localises to $\alpha(b X)$, for $b \in\left(a^{d}\right), d \gg 0$.

Proof: Let $\alpha(X)=\prod_{k=1}^{r} g e_{i_{k} j_{k}}\left(f_{k}(X)\right)$, where $f_{k}(X)=f_{k}(0)+X g_{k}(X)$. Therefore, $\alpha(X)=\prod_{k=1}^{r} \gamma_{k} g e_{i_{k} j_{k}}\left(X g_{k}(X)\right) \gamma_{k}^{-1}$, where $\gamma_{l}=\prod_{k=1}^{l} g e_{i_{k} j_{k}}\left(f_{k}(0)\right)$ and $\gamma_{l}$ is in $\mathrm{E}^{1}\left(n, R_{a}, I_{a}\right)$. We can write $\left.\alpha\left(Y^{4^{r}} X\right)=\prod_{k=1}^{r}\left(\prod_{t=1}^{s} g e_{i_{t} j_{t}}\left(Y h_{t}(X, Y)\right) / a^{d^{\prime}}\right)\right)$, where either $i_{t}=1$ or $j_{t}=1$ (see Lemma 3.1). Note that $h_{t}(X, Y) \in R[X, Y]$, when $i_{t}=1$ and $h_{t}(X, Y) \in I[X, Y]$, when $j_{t}=1$, and $d^{\prime}$ is a natural number. Let us choose $d=d^{\prime}$ and define $\alpha^{*}(X, Y)$ to be $\prod_{k=1}^{r}\left(\prod_{t=1}^{s} g e_{i_{t} j_{t}}\left(Y h_{t}\left(X, a^{d} Y\right)\right)\right)$.

Clearly $\alpha^{*}(X, Y) \in \mathrm{E}^{1}(n, R[X, Y], I[X, Y])$ and $\alpha\left(\left(a^{d} Y\right)^{4^{r}} X\right)=\alpha^{*}(X, Y)$. Substituting $Y=1$, we get $\alpha(b X)=\alpha^{*}(X)$, for $b \in\left(a^{d}\right), d \gg 0$. Note that $\alpha^{*}(X) \in$ $\mathrm{E}^{1}(n, R[X], I[X])$, with $\alpha^{*}(0)=I d$.

Theorem 3.5. Let $R$ be a commutative ring with $R=2 R$, and let $I$ be an ideal of $R$. Let $n \geq 3$ in the linear case and $n \geq 4$ in the symplectic case. Let a be a non-nilpotent element in $R$ and $\alpha(X)$ be in $\mathrm{E}\left(n, R_{a}[X], I_{a}[X]\right)$, with $\alpha(0)=I d$. Then there exists $\alpha^{*}(X) \in \mathrm{E}(n, R[X], I[X])$, with $\alpha^{*}(0)=I d$., such that $\alpha^{*}(X)$ localises to $\alpha(b X)$, for $b \in\left(a^{d}\right), d \gg 0$.

Proof: Follows from the previous lemma and Lemma2.10, which says that $\mathrm{E}(n, R, I)$ is $\mathrm{E}^{1}(n, R, I) \cap \mathrm{G}(n, R, I)$. 


\section{Transvection Groups}

Following H. Bass one defines a transvection of a finitely generated $R$-module as follows:

Definition 4.1. Let $M$ be a finitely generated $R$-module. Let $q \in M$ and $\pi \in M^{*}=$ $\operatorname{Hom}(M, R)$, with $\pi(q)=0$. Let $\pi_{q}(p):=\pi(p) q$. An automorphism of the form $1+\pi_{q}$ is called a transvection of $M$, if either $q \in \operatorname{Um}(M)$ or $\pi \in \operatorname{Um}\left(M^{*}\right)$. Collection of transvections of $M$ is denoted by $\operatorname{Trans}(M)$. This forms a subgroup of $\operatorname{Aut}(M)$.

Definition 4.2. Let $M$ be a finitely generated $R$ module. The automorphisms of $N=(R \oplus M)$ of the form

$$
(a, p) \mapsto(a, p+a x)
$$

or of the form

$$
(a, p) \mapsto(a+\tau(p), p),
$$

where $x \in M$ and $\tau \in M^{*}$ are called elementary transvections of $N$. Let us denote the first automorphism by $E_{x}$ and the second one by $E_{\tau}^{*}$. It can be verified that these are transvections of $N$. Indeed, let us consider $\pi(t, y)=t, q=(0, x)$ to get $E_{x}$, and consider $\pi(a, p)=\tau(p)$, where $\tau \in M^{*}, q=(1,0)$ to get $E_{\tau}^{*}$. The subgroup of $\operatorname{Trans}(N)$ generated by elementary transvections is denoted by $\operatorname{ETrans}(N)$.

Definition 4.3. Let $I$ be an ideal of $R$. The group of relative transvections w.r.t. an ideal $I$ is generated by the transvections of the form $1+\pi_{q}$, where either $q \in I M$ or $\pi \in I M^{*}$. The group generated by relative transvections is denoted by $\operatorname{Trans}(M, I M)$.

Definition 4.4. Let $I$ be an ideal of $R$. The elementary transvections of $N=$ $(R \oplus M)$ of the form $E_{x}, E_{\tau}^{*}$, where $x \in I M$ and $\tau \in I M^{*}$ are called relative elementary transvections w.r.t. an ideal $I$, and the group generated by them is denoted by $\operatorname{ETrans}(I N)$. The normal closure of $\operatorname{ETrans}(I N)$ in $\operatorname{ETrans}(N)$ is denoted by $\operatorname{ETrans}(N, I N)$.

Lemma 4.5. Let $I$ be an ideal of $R$ and $M$ be a free $R$ module of rank $n \geq 2$, and $N=(R \oplus M)$. Then $\operatorname{ETrans}(N, I N)=\operatorname{Trans}(N, I N)=\mathrm{E}_{n+1}(R, I)$.

Proof: Let $I_{n+1}$ denote the identity matrix of size $n+1$. Note that when $M$ is a free $R$ module, an element of $\operatorname{Trans}(N, I N)$ looks like $I_{n+1}+v^{t} w$, for some $v, w \in R^{n+1}$. Among $v$ and $w$, one of them is unimodular and the other belongs to $I^{n+1}\left(\subseteq R^{n+1}\right)$. Also, note that $\langle v, w\rangle=v w^{t}=0$. Therefore $\operatorname{Trans}(N, I N) \subseteq \mathrm{E}_{n+1}(R, I)$ (follows from [17, Corollary 1.2 and Lemma 1.3).

Using definition of $\operatorname{ETrans}(N, I N), \mathrm{E}_{n+1}(R, I)$ and the fact that in the free case elementary transvections $E_{x}$ and $E_{\tau}^{*}$ of $N$ are of the form $\left(\begin{array}{ll}1 & x \\ 0 & I_{n}\end{array}\right)$, and $\left(\begin{array}{cc}1 & 0 \\ y^{t} & I_{n}\end{array}\right)$, respectively, we get $\operatorname{ETrans}(N, I N) \subseteq \mathrm{E}_{n+1}(R, I)$. By ([10], Lemma 2.2) and ([16], Lemma $2.7(\mathrm{a}))$ we have $\mathrm{E}_{n+1}(R, I) \subseteq \operatorname{ETrans}(N, I N)$, hence $\operatorname{ETrans}(N, I N)=\mathrm{E}_{n+1}(R, I)$. We have $\mathrm{E}_{n+1}(R, I)=\operatorname{ETrans}(N, I N) \subseteq \operatorname{Trans}(N, I N) \subseteq \mathrm{E}_{n+1}(R, I)$, and hence the result follows. 
We now establish dilation principle for the elementary transvection group in the relative case w.r.t. an ideal of the ring. Dilation principle in the absolute case is proved in ([2], Proposition 3.1).

Lemma 4.6. Let $I$ be an ideal of $R$ and $M$ be a finitely generated module of $R$. Suppose that $a$ is a non-nilpotent element of $R$ such that $M_{a}$ is a free $R_{a}$-module of rank $n \geq 2$. Let $N=(R \oplus M)$. Let $\alpha(X) \in \operatorname{Aut}(N[X])$ with $\alpha(0)=I d$., and $\alpha(X)_{a} \in \operatorname{ETrans}\left(N_{a}[X], I N_{a}[X]\right)$. Then there exists $\alpha^{*}(X) \in \operatorname{ETrans}(N[X], I N[X])$, with $\alpha^{*}(0)=I d$., such that $\alpha^{*}(X)$ localises to $\alpha(b X)$, for $b \in\left(a^{d}\right), d \gg 0$.

Proof: Using previous lemma we get $\operatorname{ETrans}\left(N_{a}[X], I N_{a}[X]\right)=\mathrm{E}_{n+1}\left(R_{a}[X], I_{a}[X]\right)$ and using Lemma2.10 we get $\mathrm{E}_{n+1}\left(R_{a}[X], I_{a}[X]\right)=\mathrm{E}_{n+1}^{1}\left(R_{a}[X], I_{a}[X]\right) \cap G L_{n+1}\left(R_{a}, I_{a}\right)$. Therefore, one can write $\alpha(X)_{a}=\prod_{t} \gamma_{t} E_{i_{t} j_{t}}\left(X f_{t}(X)\right) \gamma_{t}^{-1}$, where either $i_{t}=1$ or $j_{t}=1$, and $\gamma_{t} \in E_{n+1}^{1}\left(R_{a}, I_{a}\right)$. Note that $f_{t}(X) \in R_{a}[X]$ if $i_{t}=1$, and $f_{t}(X) \in I_{a}[X]$ if $j_{t}=1$. Using Lemma 3.1 we write

$$
\alpha\left(Y^{4^{r}} X\right)_{a}=\prod_{k} E_{i_{k} j_{k}}\left(Y h_{k}(X, Y) / a^{m}\right)
$$

where either $i_{k}=1$ or $j_{k}=1$. Note that $h_{k}(X, Y) \in R[X, Y]$ if $i_{k}=1$, and $h_{k}(X, Y) \in I[X, Y]$ if $j_{k}=1$.

Since $N_{a}$ is a free $R_{a}$ module we have $N_{a} \cong R_{a}^{n+1} \cong N_{a}^{*}$. Let $p_{1}, \ldots, p_{n+1}$ be the standard basis of $N_{a}, p_{1}^{*}, \ldots, p_{n+1}^{*}$ be the standard basis of $N_{a}^{*}$, and $e_{1}, \ldots, e_{n+1}$ be the standard basis of $R_{a}^{n+1}$. Let $s \geq 0$ be an integer such that $\widetilde{p}_{i}=a^{s} p_{i} \in N$, and $\widetilde{p_{i}^{*}}=a^{s} p_{i}^{*} \in N^{*}$, for all $i$. Note that

$$
\begin{aligned}
\alpha\left(Y^{4^{r}} X\right)_{a} & =\prod_{k}\left(I d .+\left(Y h_{k}(X, Y) / a^{m}\right) e_{i_{k}}^{t} \cdot e_{j_{k}}\right) \\
& =\prod_{k}\left(I d .+\left(Y h_{k}(X, Y) / a^{m}\right) p_{i_{k}}^{*} \cdot p_{j_{k}}\right)
\end{aligned}
$$

Let $m \geq 0$ be the maximum power of $a$ appearing in the denominators of each $h_{k}$. Let $d^{\prime}=2 s+m$. Now,

$$
\begin{aligned}
\alpha\left(\left(a^{d^{\prime}} Y\right)^{4^{r}} X\right)_{a} & \left.=\prod_{k}\left(I d .+a^{2 s} Y h_{k}\left(X, a^{d^{\prime}} Y\right)\right) p_{i_{k}}^{*} \cdot p_{j_{k}}\right) \\
& \left.=\prod_{k}\left(I d .+Y h_{k}\left(X, a^{d^{\prime}} Y\right)\right) a^{s} p_{i_{k}}^{*} \cdot a^{s} p_{j_{k}}\right) .
\end{aligned}
$$

Substituting $Y=1$ we get $\alpha\left(a^{d} X\right)_{a}=\prod_{k}\left(I d .+h_{k}^{\prime}(X) a^{s} p_{i_{k}}^{*} \cdot a^{s} p_{j_{k}}\right)$. Let us set $\alpha^{*}(X)=\prod_{k}\left(I d .+h_{k}^{\prime}(X) \widetilde{p_{i_{k}}^{*}} \cdot \widetilde{p_{j_{k}}}\right)$, where either $i_{k}=1$ or $j_{k}=1$. Note that $\alpha^{*}(X)$ belongs to $\operatorname{ETrans}(N[X]) \cap \operatorname{Aut}(N[X], I N[X]$, where $\operatorname{Aut}(N[X], I N[X]=$ $\operatorname{ker}(\operatorname{Aut}(N[X]) \longrightarrow \operatorname{Aut}(N[X] / I N[X]))$. Generators of $\operatorname{ETrans}(N[X])$ have the splitting property as $E_{x+y}=E_{x} E_{y}$ and $E_{\tau+\rho}^{*}=E_{\tau}^{*} E_{\rho}^{*}$. Therefore, using argument similar to Lemma 2.10 we can show that $\alpha^{*}(X) \in \operatorname{ETrans}(N[X], I N[X])$. It is clear from the construction that $\alpha^{*}(0)=I d$. and $\alpha^{*}(X)$ localises to $\alpha(b X)$, for $b \in\left(a^{d}\right), d \gg 0$. 
Lemma 4.7. Let $I$ be an ideal of $R$ and let $M$ be a finitely generated projective $R$ module of rank $n \geq 2$. Let $N=(R \oplus M)$. Let $\alpha(X) \in \operatorname{Aut}(N[X])$, with $\alpha(0)=I d$. If for each maximal ideal $\mathfrak{m}$ of $R, \alpha(X)_{\mathfrak{m}} \in \operatorname{ETrans}\left(N_{\mathfrak{m}}[X], I N_{\mathfrak{m}}[X]\right)$, then $\alpha(X) \in$ $\operatorname{ETrans}(N[X], I N[X])$.

Proof: One can suitably choose an element $a_{\mathfrak{m}}$ from $R \backslash \mathfrak{m}$ such that $\alpha(X)_{a_{\mathfrak{m}}}$ belongs to $\operatorname{ETrans}\left(N_{a_{\mathrm{m}}}[X], I N_{a_{\mathrm{m}}}[X]\right)$. Let us set $\gamma(X, Y)=\alpha(X+Y) \alpha(Y)^{-1}$. Note that $\gamma(X, Y)_{a_{\mathfrak{m}}}$ belongs to $\operatorname{ETrans}\left(N_{a_{\mathfrak{m}}}[X, Y], I N_{a_{\mathfrak{m}}}[X, Y]\right)$, and $\gamma(0, Y)=I d$. From Lemma 4.6 it follows that $\gamma\left(b_{\mathfrak{m}} X, Y\right) \in \operatorname{ETrans}(N[X, Y], I N[X, Y])$, for $b_{\mathfrak{m}} \in\left(a_{\mathfrak{m}}^{d}\right)$, where $d \gg 0$. Note that the ideal generated by $a_{\mathfrak{m}}^{d}$ 's is the whole ring $R$. Therefore, $c_{1} a_{\mathfrak{m}_{1}}^{d}+\ldots+c_{k} a_{\mathfrak{m}_{k}}^{d}=1$, for some $c_{i} \in R$. Let $b_{m_{i}}=c_{i} a_{m_{i}}^{d} \in\left(a_{m_{i}}^{d}\right)$. It is easy to see that $\alpha(X)=\prod_{i=1}^{k-1} \gamma\left(b_{m_{i}} X, T\right) \gamma\left(b_{m_{k}} X, 0\right)$, where $T_{i}=b_{m_{i+1}} X+\cdots+b_{m_{k}} X$. Each term in the right hand side of this expression belongs to $\operatorname{ETrans}(N[X], I N[X])$ and hence $\alpha(X) \in \operatorname{ETrans}(N[X], I N[X])$.

Theorem 4.8. Let $I$ be an ideal of $R$ and let $M$ be a finitely generated projective $R$-module of rank $n \geq 2$. Let $N=(R \oplus M)$. Let $q(X) \in \operatorname{Um}(N[X], I N[X])$, where $q(X)$ is of the form $(a(X), p(X))$. If for each maximal ideal $\mathfrak{m}$ of $R, q(X) \in$ $q(0) \operatorname{ETrans}\left(N_{\mathfrak{m}}[X], I N_{\mathfrak{m}}[X]\right)$, then $q(X) \in q(0) \operatorname{ETrans}(N[X], I N[X])$.

Proof: For each maximal ideal $\mathfrak{m}$ of $R$, we get $\beta_{(\mathfrak{m})}(X)$ in $\operatorname{ETrans}\left(N_{\mathfrak{m}}[X], I N_{\mathfrak{m}}[X]\right)$ such that $q(X) \beta_{(\mathfrak{m})}(X)=q(0)$. Let us set $\gamma(X, T)=\beta_{(\mathfrak{m})}(X+T) \beta_{(\mathfrak{m})}(X)^{-1}$. Clearly $\gamma(X, T) \in \operatorname{ETrans}\left(N_{\mathfrak{m}}[X, T], I N_{\mathfrak{m}}[X, T]\right)$. Since there are only finitely many denominators involved in the expression of $\gamma(X, T)$, there exists $a_{\mathfrak{m}} \in R \backslash \mathfrak{m}$ such that $\gamma(X, T)$ belongs to ETrans $\left(N_{a_{\mathfrak{m}}}[X, T], I N_{a_{\mathfrak{m}}}[X, T]\right)$ and $\gamma(X, 0)=I d$. Using Lemma4.6 it follows that $\gamma\left(X, b_{\mathfrak{m}} T\right) \in \operatorname{ETrans}(N[X, T], I N[X, T])$ for $b_{\mathfrak{m}} \in\left(a_{\mathfrak{m}}^{d}\right), d \gg 0$. We have $q\left(X+b_{\mathfrak{m}} T\right) \gamma\left(X, b_{\mathfrak{m}} T\right)=q\left(X+b_{\mathfrak{m}} T\right) \beta_{(\mathfrak{m})}\left(X+b_{\mathfrak{m}} T\right) \beta_{(\mathfrak{m})}(X)^{-1}=q(0) \beta_{(\mathfrak{m})}(X)^{-1}=$ $q(X)$.

Note that the ideal generated by $a_{\mathfrak{m}}^{d}$ 's is the whole ring $R$. Therefore $c_{1} a_{\mathfrak{m}_{1}}^{d}+$ $\cdots+c_{k} a_{\mathfrak{m}_{k}}^{d}=1$, for some $c_{i} \in R$. Let $b_{m_{i}}=c_{i} a_{m_{i}}^{d} \in\left(a_{m_{i}}^{d}\right)$. In the above equation replacing $X$ by $b_{\mathfrak{m}_{2}} X+\cdots+b_{\mathfrak{m}_{k}} X$ and replacing $b_{m} T$ by $b_{m_{1}} X$ we get,

$q(X)=q\left(b_{\mathfrak{m}_{1}} X+b_{\mathfrak{m}_{2}} X+\cdots+b_{\mathfrak{m}_{k}} X\right) \in q\left(b_{\mathfrak{m}_{2}} X+\cdots+b_{\mathfrak{m}_{k}} X\right) \operatorname{ETrans}(N[X], I N[X])$.

Again, in the above equation replacing $X$ by $b_{\mathfrak{m}_{3}} X+\cdots+b_{\mathfrak{m}_{k}} X$ and replacing $b_{\mathfrak{m}} T$ by $b_{\mathfrak{m}_{2}} X$ we get, $q\left(b_{\mathfrak{m}_{2}} X+\cdots+b_{\mathfrak{m}_{k}} X\right) \in q\left(b_{\mathfrak{m}_{3}} X+\cdots+b_{\mathfrak{m}_{k}} X\right) \operatorname{ETrans}(N[X], I N[X])$. Continuing in this way we get $q\left(b_{m_{k}} X+0\right) \in q(0) \operatorname{ETrans}(N[X], I N[X])$. Combining all these we get $q(X) \prod_{i=1}^{k-1} \gamma\left(b_{\mathfrak{m}_{i+1}} X+\cdots+b_{\mathfrak{m}_{k}} X, b_{\mathfrak{m}_{i}} X\right) \gamma\left(0, b_{\mathfrak{m}_{k}} X\right)=q(0)$, where $\prod_{i=1}^{k-1} \gamma\left(b_{\mathfrak{m}_{i+1}} X+\cdots+b_{\mathfrak{m}_{k}} X, b_{\mathfrak{m}_{i}} X\right) \gamma\left(0, b_{\mathfrak{m}_{k}} X\right) \in \operatorname{ETrans}(N[X], I N[X])$.

Before we establish equality of the transvection group and elementary transvection group, we prove a lemma to show that every transvection is homotopic to identity. Note that for any abelian group $G$, we understand by $G[X]$ the abelian group of all polynomials in $X$ with coefficients in $G$.

Lemma 4.9. Let $M$ be a finitely generated $R$-module and $\alpha \in \operatorname{Trans}(M)$. Then there exists $\beta(X) \in \operatorname{Trans}(M[X])$ such that $\beta(1)=\alpha$ and $\beta(0)=I d$. 
Proof: As $\alpha \in \operatorname{Trans}(M)$, it is product of elements of the form $I d+\pi_{q}$, where $\pi \in M^{*}, q \in M$ with $\pi(q)=0$. Here either $\pi$ or $q$ is unimodular. Let $\pi X$ represent $\pi$ times $X$ which belongs to $M^{*}[X]$ and $q X$ represent $q$ times $X$ which belongs to $M[X]$. Define $\pi X(q)=\pi(q) X$ or $\pi(q X)=\pi(q) X$. We set $\beta(X)$ to be the product of elements of the form $I d+\pi_{q X}$ or $I d+\pi X_{q}$, whenever $I d+\pi_{q}$ appears in the expression of $\alpha$. This choice depends on whether $\pi$ is unimodular or $q$ is unimodular. Then $\beta(1)=\alpha$ and $\beta(0)=I d$.

Proposition 4.10. Let $I$ be an ideal of $R$. Let $M$ be a finitely generated projective $R$ module of rank at least 2, and $N=(R \oplus M)$. Then $\operatorname{Trans}(N, I N)=\operatorname{ETrans}(N, I N)$.

Proof: Note that $\operatorname{ETrans}(N, I N) \subseteq \operatorname{Trans}(N, I N)$. Let us consider an element $\alpha \in \operatorname{Trans}(N, I N)$. By Lemma 4.9 there exists $\alpha(X) \in \operatorname{Trans}(N[X], I N[X])$ such that $\alpha(1)=\alpha$ and $\alpha(0)=I d$. Let $\mathfrak{m}$ be a maximal ideal of $R$. We have $\alpha(X)_{\mathfrak{m}} \in$ $\operatorname{Trans}\left(N_{\mathfrak{m}}[X], I N_{\mathfrak{m}}[X]\right)=\operatorname{ETrans}\left(N_{\mathfrak{m}}[X], I N_{\mathfrak{m}}[X]\right)$ (see Lemma 4.5). This is true for all maximal ideal $\mathfrak{m}$ of $R$ and hence $\alpha(X) \in \operatorname{ETrans}(N[X], I N[X])$ by Lemma 4.7. Substituting $X=1$ we get $\alpha \in \operatorname{ETrans}(N, I N)$. Therefore $\operatorname{Trans}(N, I N) \subseteq$ $\operatorname{ETrans}(N, I N)$.

\section{Symplectic Modules and Symplectic Transvections}

Definition 5.1. Alternating Matrix: A matrix in $\mathrm{M}_{n}(R)$ is said to be alternating if it has the form $\nu-\nu^{t}$, where $\nu \in \mathrm{M}_{n}(R)$. (It follows that its diagonal elements are zeros.)

Lemma 5.2. Let $(R, \mathfrak{m})$ be a local ring and $I$ be an ideal of $R$. Let $\varphi$ be an alternating matrix of Pfaffian 1 over $R$, and $\varphi \equiv \psi_{n}(\bmod I)$. Then $\varphi$ is of the form

$$
(1 \perp \varepsilon)^{t} \psi_{n}(1 \perp \varepsilon),
$$

for some $\varepsilon \in \mathrm{E}_{2 n-1}(R, I)$.

Proof: We will prove the result using induction on $n$. When $\varphi$ is of size $2 \times 2$, the result is true. Let us assume that the result is true for alternating matrix of size $2(n-1) \times 2(n-1)$, i.e, for any $2(n-1) \times 2(n-1)$ alternating matrix $\varphi^{*}$ of Pfaffian 1 with $\varphi^{*} \equiv \psi_{n-1}(\bmod I)$, we have $\eta \in \mathrm{E}_{2 n-3}(R, I)$ such that $\varphi^{*}=(1 \perp \eta)^{t} \psi_{n-1}(1 \perp \eta)$.

We will prove the result for alternating matrix $\varphi$ of size $2 n \times 2 n$. Let $\varphi=\left(\begin{array}{cc}0 & a \\ -a^{t} & \alpha\end{array}\right) \equiv$ $\psi_{n}(\bmod I)$, where $a \in \operatorname{Um}_{2 n-1}(R, I)$ and $\alpha$ is alternating matrix of size $(2 n-1) \times$ $(2 n-1)$. Note that $\alpha \equiv\left(\begin{array}{cc}0 & 0 \\ 0 & \psi_{n-1}\end{array}\right)(\bmod I)$.

As $R$ is local ring we have $a=e_{1} \beta$, where $\beta \in \mathrm{E}_{2 n-1}(R, I)$ (see Remark 2.4). Hence $\left(1 \perp \beta^{t}\right)^{-1} \varphi(1 \perp \beta)^{-1}=\left(\begin{array}{cc}0 & e_{1} \\ -e_{1}^{t} & \gamma\end{array}\right)$, where $\gamma=\left(\beta^{t}\right)^{-1} \alpha \beta^{-1}$. Note that $\gamma$ is an alternating matrix. Therefore $\gamma$ can be written as $\left(\begin{array}{cc}0 & b \\ -b^{t} & \varphi^{*}\end{array}\right)$. Note that $\bar{\gamma}=\left(\bar{\beta}^{t}\right)^{-1} \bar{\alpha} \bar{\beta}^{-1} \equiv\left(\begin{array}{cc}0 & 0 \\ 0 & \psi_{n-1}\end{array}\right)(\bmod I)$, and hence $b \in I^{2 n-2}$ and $\varphi^{*} \equiv \psi_{n-1}(\bmod I)$. Now $\left(\begin{array}{ccc}1 & 0 & 0 \\ 0 & 1 & -b \varphi^{*-1} \\ 0 & 0 & I_{2 n-2}\end{array}\right)\left(1 \perp \beta^{t}\right)^{-1} \varphi(1 \perp \beta)^{-1}\left(\begin{array}{ccc}1 & 0 & 0 \\ 0 & 1 & -b \varphi^{*-1} \\ 0 & 0 & I_{2 n-2}\end{array}\right)^{t}=\left(\begin{array}{ccc}0 & 1 & 0 \\ -1 & 0 & 0 \\ 0 & 0 & \varphi^{*}\end{array}\right)$. 
Let us call the matrix $\left(\left(I_{3} \perp \eta\right)^{-1}\right)^{t}\left(\begin{array}{ccc}1 & 0 & 0 \\ 0 & 1 & -b \varphi^{*-1} \\ 0 & 0 & I_{2 n-2}\end{array}\right)\left(1 \perp \beta^{t}\right)^{-1}=\left((1 \perp \varepsilon)^{-1}\right)^{t}$. Note that $\varepsilon \in \mathrm{E}_{2 n-1}(R, I)$. Using induction hypothesis we get $\left((1 \perp \varepsilon)^{-1}\right)^{t} \varphi(1 \perp \varepsilon)^{-1}=$ $\left(\left(I_{3} \perp \eta\right)^{-1}\right)^{t}\left(\begin{array}{ccc}0 & 1 & 0 \\ -1 & 0 & 0 \\ 0 & 0 & \varphi^{*}\end{array}\right)\left(I_{3} \perp \eta\right)^{-1}=\psi_{n}$, and hence $\varphi=(1 \perp \varepsilon)^{t} \psi_{n}(1 \perp \varepsilon)$. Therefore the result is established.

Remark 5.3. The condition that the alternating matrices are of Pfaffian one can be replaced by the weaker condition that the alternating matrix be invertible, and congruent to $\left(u \psi_{1} \perp \psi_{n-1}\right)(\bmod I)$, where $u=$ Pfaffian $\varphi$, is of the form $(1 \perp$ $E)^{t}\left(u \psi_{1} \perp \psi_{n-1}\right)(1 \perp E)$, for some relative elementary matrix $E$.

Remark 5.4. Let $\varphi$ be an alternating matrix of Pfaffian 1 , over $R$. Let $\mathfrak{m}$ be a maximal ideal of $R$ and $R_{\mathfrak{m}}$ be the local ring at $\mathfrak{m}$. We will get $\varepsilon(\mathfrak{m}) \in \mathrm{E}_{2 n-1}\left(R_{\mathfrak{m}}\right)$ such that over $R_{\mathfrak{m}}$ we have $\varphi=(1 \perp \varepsilon(\mathfrak{m}))^{t} \psi_{n}(1 \perp \varepsilon(\mathfrak{m}))$. Let a be the product of denominators of all the entries of $\varepsilon(\mathfrak{m})$. Clearly a is not in $\mathfrak{m}$. Hence we get $\varepsilon$ from $\mathrm{E}_{2 n-1}\left(R_{a}\right)$ such that $\varphi=(1 \perp \varepsilon)^{t} \psi_{n}(1 \perp \varepsilon)$.

Note: When dealing with relative case w.r.t. an ideal $I$ of $R$, we will always assume that the alternating matrix $\varphi$ of Pfaffian 1 is congruent to $\psi_{n}(\bmod I)$. Using Lemma 5.2 and arguing as above we get that over ring $R_{\mathfrak{m}}$ we have $\varphi=(1 \perp \varepsilon)^{t} \psi_{n}(1 \perp \varepsilon)$, where $\varepsilon \in \mathrm{E}_{2 n-1}\left(R_{a}, I_{a}\right)$, for some $a \notin \mathfrak{m}$. We will constantly use this fact without even referring to it!

Definition 5.5. A symplectic $R$-module is a pair $(P,\langle\rangle$,$) , where P$ is a finitely generated projective $R$-module of even rank and $\langle\rangle:, P \times P \longrightarrow R$ is a non-degenerate (i.e, $P \cong P^{*}$ by $x \longrightarrow\langle x,-\rangle$ ) alternating bilinear form.

Definition 5.6. Let $\left(P_{1},\langle,\rangle_{1}\right)$ and $\left(P_{2},\langle,\rangle_{2}\right)$ be two symplectic $R$-modules. Their orthogonal sum is the pair $(P,\langle\rangle$,$) , where P=P_{1} \oplus P_{2}$ and the inner product is defined by $\left\langle\left(p_{1}, p_{2}\right),\left(q_{1}, q_{2}\right)\right\rangle=\left\langle p_{1}, q_{1}\right\rangle_{1}+\left\langle p_{2}, q_{2}\right\rangle_{2}$.

There is a non-degenerate bilinear form $\langle$,$\rangle on the R$-module $\mathbb{H}(R)=R \oplus R^{*}$, namely $\left\langle\left(a_{1}, f_{1}\right),\left(a_{2}, f_{2}\right)\right\rangle=f_{2}\left(a_{1}\right)-f_{1}\left(a_{2}\right)$.

Definition 5.7. An isometry of a symplectic module $(P,\langle\rangle$,$) is an automorphism$ of $P$ which fixes the bilinear form. The group of isometries of $(P,\langle\rangle$,$) is denoted by$ $\operatorname{Sp}(P,\langle\rangle$,$) .$

Definition 5.8. In [5] Bass has defined a symplectic transvection of a symplectic module $P$ to be an automorphism of the form

$$
\sigma(p)=p+\langle u, p\rangle v+\langle v, p\rangle u+\alpha\langle u, p\rangle u,
$$

where $\alpha \in R, u, v \in P$ are fixed elements with $\langle u, v\rangle=0$, and either $u$ or $v$ is unimodular. It is easy to check that $\langle\sigma(p), \sigma(q)\rangle=\langle p, q\rangle$ and $\sigma$ has an inverse $\tau(p)=$ $p-\langle u, p\rangle v-\langle v, p\rangle u-\alpha\langle u, p\rangle u$.

The subgroup of $\operatorname{Sp}(P,\langle\rangle$,$) generated by the symplectic transvections is denoted$ by $\operatorname{Transsp}_{\mathrm{Sp}}(P,\langle\rangle$,$) (see [19], Page 35).$

Now onwards $Q$ will denote $\left(R^{2} \oplus P\right)$ with induced form on $(\mathbb{H}(R) \oplus P)$, and $Q[X]$ will denote $\left(R[X]^{2} \oplus P[X]\right)$ with induced form on $(\mathbb{H}(R[X]) \oplus P[X])$. 
Definition 5.9. The symplectic transvections of $Q=\left(R^{2} \oplus P\right)$ of the form

$$
(a, b, p) \mapsto(a, b-\langle p, q\rangle-\alpha a, p-a q),
$$

or of the form

$$
(a, b, p) \mapsto(a+\langle p, q\rangle+\beta b, b, p-b q),
$$

where $\alpha, \beta \in R$ and $q \in P$, are called elementary symplectic transvections. Let us denote the first isometry by $\rho(q, \alpha)$ and the second one by $\mu(q, \beta)$. It can be verified that the elementary symplectic transvections are symplectic transvections on $Q$. Indeed, consider $(u, v)=((0,1,0),(0,0, q))$ to get $\rho(q, \alpha)$ and consider $(u, v)=$ $((-1,0,0),(0,0, q))$ to get $\mu(q, \beta)$.

The subgroup of $\operatorname{Transsp}_{\mathrm{S}}(Q,\langle\rangle$,$) generated by elementary symplectic transvections$ is denoted by ETrans ${ }_{\mathrm{Sp}}(Q,\langle\rangle$,$) .$

Definition 5.10. Let $I$ be an ideal of $R$. The group of relative symplectic transvections w.r.t. an ideal $I$ is generated by the symplectic transvecions of the form $\sigma(p)=p+\langle u, p\rangle v+\langle v, p\rangle u+\alpha\langle u, p\rangle u$, where $\alpha \in I$ and $u \in P, v \in I P$ are fixed elements with $\langle u, v\rangle=0$. The group generated by relative symplectic transvections is denoted by $\operatorname{Trans}_{\mathrm{Sp}}(P, I P,\langle\rangle$,$) .$

Definition 5.11. Let $I$ be an ideal of $R$. The elementary symplectic transactions of $Q$ of the form $\rho(q, \alpha), \mu(q, \beta)$, where $q \in I P$ and $\alpha, \beta \in I$ are called relative elementary symplectic transvections w.r.t. an ideal $I$.

The subgroup of $\operatorname{ETrans}_{\mathrm{Sp}}(Q,\langle\rangle$,$) generated by relative elementary symplectic$ transvections is denoted by ETranssp $(I Q,\langle\rangle$,$) . The normal closure of \operatorname{ETrans}_{\mathrm{Sp}}(I Q,\langle\rangle$, in $\operatorname{ETranssp}_{\mathrm{s}}(Q,\langle\rangle$,$) is denoted by \operatorname{ETrans}_{\mathrm{Sp}}(Q, I Q,\langle\rangle$,$) .$

Remark 5.12. Let $P=\oplus_{i=1}^{2 n} R e_{i}$ be a free $R$-module. The non-degenerate alternating bilinear form $\langle$,$\rangle on P$ corresponds to an alternating matrix $\varphi$ with Praffian 1 with respect to the basis $\left\{e_{1}, e_{2}, \ldots, e_{2 n}\right\}$ of $P$ and we write $\langle p, q\rangle=p \varphi q^{t}$.

In this case the symplectic transvection $\sigma(p)=p+\langle u, p\rangle v+\langle v, p\rangle u+\alpha\langle u, p\rangle u$ corresponds to the matrix $\left(I_{2 n}-\varphi u^{t} v-\varphi v^{t} u\right)\left(I_{2 n}-\alpha \varphi u^{t} u\right)$ and the group generated by them is denoted by $\operatorname{Trans}_{\mathrm{Sp}}\left(P,\langle,\rangle_{\varphi}\right)$.

Also in this case ETranssp $\left(Q,\langle,\rangle_{\psi_{1} \perp \varphi}\right)$ will be generated by the matrices of the form $\rho_{\varphi}(q, \alpha)=\left(\begin{array}{ccc}1 & -\alpha & -q \\ 0 & 1 & 0 \\ 0 & -\varphi q^{t} & I_{2 n}\end{array}\right)$, and $\mu_{\varphi}(q, \beta)=\left(\begin{array}{ccc}1 & 0 & 0 \\ \varphi q^{t} & 0 & -q \\ I_{2 n}\end{array}\right)$.

Note that for $q=\left(q_{1}, \ldots, q_{2 n}\right) \in R^{2 n}$, and for the standard alternating matrix $\psi_{n}$, we have

$$
\begin{aligned}
\rho_{\psi_{n}}(q, \alpha) & =s e_{12}(-\alpha) \prod_{i=3}^{2 n+2} s e_{1 i}\left(-q_{i-2}\right) \\
\mu_{\psi_{n}}(q, \beta) & =s e_{21}(\beta) \prod_{i=3}^{2 n+2} s e_{i 1}\left((-1)^{i+1} q_{\sigma(i-2)}\right) .
\end{aligned}
$$


We shall implicitly use the assumptions and notations in the statement of Remark 5.12 in the sequel below.

Lemma 5.13. Let $R$ be a commutative ring and $I$ be an ideal of $R$. Let $P$ be a free $R$-module of rank $2 n, n \geq 1$. If $\varphi=\psi_{n}$, the standard alternating matrix, then $\operatorname{Trans}_{\mathrm{Sp}}\left(Q, I Q,\langle,\rangle_{\psi_{n+1}}\right)=\operatorname{ESp}_{2 n+2}(R, I)$.

Proof: For proof see ([1], Lemma 1.10).

Lemma 5.14. Let $R$ be a commutative ring with $R=2 R$, and let $I$ be an ideal of $R$. Let $P$ be a free $R$-module of rank $2 n, n \geq 1$. If $\varphi=\psi_{n}$, the standard alternating matrix, then $\operatorname{ETranssp}_{\mathrm{S}}\left(Q, I Q,\langle,\rangle_{\psi_{n+1}}\right)=\operatorname{ESp}_{2 n+2}(R, I)$.

Proof: We first show $\operatorname{ETrans}_{\mathrm{Sp}}\left(Q, I Q,\langle,\rangle_{\psi_{n+1}}\right)$ is a subset of $\operatorname{ESp}_{2 n+2}(R, I)$. It is enough to show that an element of the form $s_{\psi_{n}}\left(q_{1}, \alpha_{1}\right) t_{\psi_{n}}\left(q_{2}, \beta\right) s_{\psi_{n}}\left(q_{1}, \alpha\right)^{-1}$ is in $\operatorname{ESp}_{2 n}(R, I)$, for $q_{1} \in R^{2 n}, q_{2} \in I^{2 n}\left(\subseteq R^{2 n}\right), \alpha \in R$, and $\beta \in I$. Here $t_{\psi_{n}}$ and $s_{\psi_{n}}$ represents either $\rho_{\psi_{n}}$ or $\mu_{\psi_{n}}$. Using equations (11), (2) we get $\operatorname{ETrans}_{\mathrm{Sp}}\left(Q, I Q,\langle,\rangle_{\psi_{n+1}}\right) \subseteq$ $\operatorname{ESp}_{2 n}(R, I)$.

To show the other inclusion we recall the equivalent definition of the relative group which says that $\operatorname{ESp}_{2 n}(R, I)$ is the smallest normal subgroup of $\operatorname{ESp}_{2 n}(R)$ containing $s e_{21}(x)$, where $x \in I$. We have $g e_{21}(x) g^{-1} \in \operatorname{ETrans}_{\mathrm{Sp}}\left(Q, I Q,\langle,\rangle_{\psi_{n+1}}\right)$, hence $\operatorname{ESp}_{2 n}(R, I) \subseteq \operatorname{ETrans}_{\mathrm{Sp}}\left(Q, I Q,\langle,\rangle_{\psi_{n+1}}\right)$. Therefore, the equality is established.

Lemma 5.15. Let $P$ be a free $R$-module of $\operatorname{rank} 2 n$. Let $\left(P,\langle,\rangle_{\varphi}\right)$ and $\left(P,\langle,\rangle_{\varphi^{*}}\right)$ be two symplectic $R$-modules with $\varphi=(1 \perp \varepsilon)^{t} \varphi^{*}(1 \perp \varepsilon)$, for some $\varepsilon \in \mathrm{E}_{2 n-1}(R)$. Then

$$
\begin{aligned}
\operatorname{Trans}_{\mathrm{Sp}}\left(P,\langle,\rangle_{\varphi}\right) & =(1 \perp \varepsilon)^{t} \operatorname{Trans}_{\mathrm{Sp}}\left(P,\langle,\rangle_{\varphi^{*}}\right)\left((1 \perp \varepsilon)^{t}\right)^{-1}, \\
\operatorname{ETrans}_{\mathrm{Sp}}\left(Q,\langle,\rangle_{\psi_{1} \perp \varphi}\right) & =\left(I_{3} \perp \varepsilon\right)^{t} \operatorname{ETrans}_{\mathrm{Sp}}\left(Q,\langle,\rangle_{\psi_{1} \perp \varphi^{*}}\right)\left(\left(I_{3} \perp \varepsilon\right)^{t}\right)^{-1} .
\end{aligned}
$$

Proof: In the free case for symplectic transvections we have

$$
\begin{aligned}
& \left(I_{2 n}-\varphi u^{t} v-\varphi v^{t} u\right)\left(I_{2 n}-\alpha \varphi u^{t} u\right) \\
= & (1 \perp \varepsilon)^{t}\left(I_{2 n}-\varphi^{*} \tilde{u}^{t} \tilde{v}-\varphi^{*} \tilde{v}^{t} \tilde{u}\right)\left(I_{2 n}-\alpha \varphi^{*} \tilde{u}^{t} \tilde{u}\right)\left((1 \perp \varepsilon)^{t}\right)^{-1},
\end{aligned}
$$

where $\tilde{u}=u(1 \perp \varepsilon)^{t}$ and $\tilde{v}=v(1 \perp \varepsilon)^{t}$. Hence the first equality follows.

For elementary symplectic transvections we have

$$
\begin{aligned}
& \left(I_{2} \perp(1 \perp \varepsilon)\right)^{t} \rho_{\varphi^{*}}(q, \alpha)\left(\left(I_{2} \perp(1 \perp \varepsilon)\right)^{t}\right)^{-1}=\rho_{\varphi}\left(q\left((1 \perp \varepsilon)^{t}\right)^{-1}, \alpha\right), \\
& \left(I_{2} \perp(1 \perp \varepsilon)\right)^{t} \mu_{\varphi^{*}}(q, \beta)\left(\left(I_{2} \perp(1 \perp \varepsilon)\right)^{t}\right)^{-1}=\mu_{\varphi}\left(q\left((1 \perp \varepsilon)^{t}\right)^{-1}, \beta\right),
\end{aligned}
$$

hence the second equality follows.

Lemma 5.16. Let $I$ be an ideal of $R$ and $P$ be a free $R$-module of rank $2 n$. Let $\left(P,\langle,\rangle_{\varphi}\right)$ and $\left(P,\langle,\rangle_{\varphi^{*}}\right)$ be two symplectic $R$-modules with $\varphi=(1 \perp \varepsilon)^{t} \varphi^{*}(1 \perp \varepsilon)$, for some $\varepsilon \in \mathrm{E}_{2 n-1}(R, I)$. Then

$$
\begin{aligned}
\operatorname{Trans}_{\mathrm{Sp}}\left(P, I P,\langle,\rangle_{\varphi}\right) & =(1 \perp \varepsilon)^{t} \operatorname{Transsp}_{\mathrm{Sp}}\left(P, I P,\langle,\rangle_{\varphi^{*}}\right)\left((1 \perp \varepsilon)^{t}\right)^{-1}, \\
\operatorname{ETrans}_{\mathrm{Sp}}\left(Q, I Q,\langle,\rangle_{\psi_{1} \perp \varphi}\right) & =\left(I_{3} \perp \varepsilon\right)^{t} \operatorname{ETranssp}\left(Q, I Q,\langle,\rangle_{\psi_{1} \perp \varphi^{*}}\right)\left(\left(I_{3} \perp \varepsilon\right)^{t}\right)^{-1} .
\end{aligned}
$$


Proof: Using the three equations appearing in the proof of Lemma 5.15, we get these equalities.

Proposition 5.17. Let $R$ be a commutative ring with $R=2 R$, and let $I$ be an ideal of $R$. Let $\left(P,\langle,\rangle_{\varphi}\right)$ be a symplectic $R$-module with $P$ free of rank $2 n, n \geq 1$. Let $\varphi=(1 \perp \varepsilon)^{t} \psi_{n}(1 \perp \varepsilon)$, for some $\varepsilon \in \mathrm{E}_{2 n-1}(R, I)$. Then $\operatorname{Trans}_{\mathrm{Sp}}\left(Q, I Q,\langle,\rangle_{\psi_{1} \perp \varphi}\right)=$ $\operatorname{ETranssp}_{\mathrm{Sp}}\left(Q, I Q,\langle,\rangle_{\psi_{1} \perp \varphi}\right)$.

Proof: Using Lemma 5.13, Lemma 5.14, and Lemma 5.16 we get,

$$
\begin{aligned}
\operatorname{Trans}_{\mathrm{Sp}}\left(Q, I Q,\langle,\rangle_{\psi_{1} \perp \varphi}\right) & =\left(I_{3} \perp \varepsilon\right)^{t} \operatorname{Transsp}_{\mathrm{Sp}}\left(Q, I Q,\langle,\rangle_{\psi_{n+1}}\right)\left(\left(I_{3} \perp \varepsilon\right)^{t}\right)^{-1} \\
& =\left(I_{3} \perp \varepsilon\right)^{t} \mathrm{ESp}_{2+2 n}(R, I)\left(\left(I_{3} \perp \varepsilon\right)^{t}\right)^{-1}
\end{aligned}
$$

and

$$
\begin{aligned}
\operatorname{ETranssp}_{\mathrm{p}}\left(Q, I Q,\langle,\rangle_{\psi_{1} \perp \varphi}\right) & =\left(I_{3} \perp \varepsilon\right)^{t} \operatorname{ETranssp}_{\mathrm{S}}\left(Q, I Q,\langle,\rangle_{\psi_{n+1}}\right)\left(\left(I_{3} \perp \varepsilon\right)^{t}\right)^{-1} \\
& =\left(I_{3} \perp \varepsilon\right)^{t} \mathrm{ESp}_{2+2 n}(R, I)\left(\left(I_{3} \perp \varepsilon\right)^{t}\right)^{-1},
\end{aligned}
$$

and hence the equality is established.

Remark 5.18. In view of above two lemmas, for any symplectic module $\left(P,\langle,\rangle_{\varphi}\right)$ over a local ring $(R, \mathfrak{m})$, we have $\operatorname{Trans}_{\mathrm{Sp}}\left(Q, I Q,\langle,\rangle_{\psi_{1} \perp \varphi}\right)=\operatorname{ETrans}_{\mathrm{Sp}}\left(Q, I Q,\langle,\rangle_{\psi_{1} \perp \varphi}\right)$. Here $I$ is an ideal of the ring $R$.

Next we establish dilation principle for elementary symplectic transvection group.

Lemma 5.19. Let $R$ be a commutative ring with $R=2 R$, and let $I$ be an ideal of $R$. Let $(P,\langle\rangle$,$) be a symplectic R$-module with $P$ finitely generated projective $R$ module of rank $2 n, n \geq 1$. Suppose that a is a non-nilpotent element of $R$ such that $P_{a}$ is a free $R_{a}$ module and the bilinear form $\langle$,$\rangle corresponds to the alternat-$ ing matrix $\varphi$ (w.r.t. some basis). Assume that $\varphi=\left(\begin{array}{l}1 \\ \varepsilon\end{array}\right)^{t} \psi_{n}(1 \perp \varepsilon)$, for some $\varepsilon \in \mathrm{E}_{2 n-1}\left(R_{a}, I_{a}\right)$. Let $\alpha(X) \in \operatorname{Aut}(Q[X])$, with $\alpha(0)=I d$, and $\alpha(X)_{a} \in$ $\left.\operatorname{ETranssp}_{\left(Q_{a}\right.}[X], I Q_{a}[X],\langle,\rangle_{\psi_{1} \perp \varphi}\right)$. Then, there exists $\alpha^{*}(X) \in \operatorname{ETranssp}(Q[X], I Q[X],\langle\rangle$,$) ,$ with $\alpha^{*}(0)=I d$., such that $\alpha^{*}(X)$ localises to $\alpha(b X)$, for $b \in\left(a^{d}\right), d \gg 0$.

Proof: We have $P_{a} \cong R_{a}^{2 n}$. Let $e_{1}, \ldots, e_{2 n+2}$ be the standard basis of $Q_{a}$ with respect to which the bilinear form on $Q_{a}$ will correspond to $\psi_{1} \perp \varphi$. Given that $\alpha(X)_{a} \in$ $\operatorname{ETranssp}_{S}\left(Q_{a}[X], I Q_{a}[X],\langle,\rangle_{\psi_{1} \perp \varphi}\right)$ and $\left.\operatorname{ETranssp}_{\left(Q_{a}\right.}[X], I Q_{a}[X],\langle,\rangle_{\psi_{1} \perp \varphi}\right)=\left(I_{3} \perp\right.$ $\varepsilon)^{t} \operatorname{ESp}_{2 n+2}\left(R_{a}[X], I_{a}[X]\right)\left(\left(I_{3} \perp \varepsilon\right)^{t}\right)^{-1}$ by Lemma 5.14, and Lemma 5.16. Therefore, $\alpha(X)_{a}=\left(I_{3} \perp \varepsilon\right)^{t} \beta(X)\left(\left(I_{3} \perp \varepsilon\right)^{t}\right)^{-1}$, for some $\beta(X) \in \operatorname{ESp}_{2 n+2}\left(R_{a}[X], I_{a}[X]\right)$, with $\beta(0)=I d$. Note that $\operatorname{ESp}_{2 n+2}\left(R_{a}[X], I_{a}[X]\right)=\operatorname{ESp}_{2 n+2}^{1}\left(R_{a}[X], I_{a}[X]\right) \cap$ $\operatorname{Sp}_{2 n+2}\left(R_{a}[X], I_{a}[X]\right)$. Hence we can write $\beta(X)=\prod_{t} \gamma_{t} s e_{i_{t} j_{t}}\left(X f_{t}(X)\right) \gamma_{t}^{-1}$, where either $i_{t}=1$, or $j_{t}=1$, and $\gamma_{t} \in \operatorname{ESp}_{2 n+2}^{1}\left(R_{a}, I_{a}\right)$. Note that $f_{t}(X) \in R_{a}[X]$, when $i_{t}=1$ and $f_{t}(X) \in I_{a}[X]$, when $j_{t}=1$. Using Lemma 3.1 we get $\beta\left(Y^{4^{r}} X\right)=$ $\prod_{k} s e_{i_{k} j_{k}}\left(Y h_{k}(X, Y) / a^{m}\right)$, with either $i_{k}=1$ or $j_{k}=1$. Note that $h_{k}(X, Y) \in$ 
$R[X, Y]$, when $i_{k}=1$ and $h_{k}(X, Y) \in I[X, Y]$, when $j_{k}=1$. Note that

$$
\begin{aligned}
s e_{12}\left(Y h_{k}(X, Y) / a^{m}\right)= & I_{2 n+2}+\left(Y h_{k}(X, Y) / a^{m}\right) \psi_{n+1} e_{2}^{t} e_{2}, \\
s e_{1 j_{k}}\left(Y h_{k}(X, Y) / a^{m}\right)= & I_{2 n+2}+\left(Y h_{k}(X, Y) / a^{m}\right) \psi_{n+1} e_{2}^{t} e_{j_{k}} \\
& +\left(Y h_{k}(X, Y) / a^{m}\right) \psi_{n+1} e_{j_{k}}{ }^{t} e_{2}, \text { for } j_{k} \geq 3, \\
s e_{21}\left(Y h_{k}(X, Y) / a^{m}\right)= & I_{2 n+2}-\left(Y h_{k}(X, Y) / a^{m}\right) \psi_{n+1} e_{1}^{t} e_{1}, \\
s e_{i_{k} 1}\left(Y h_{k}(X, Y) / a^{m}\right)= & I_{2 n+2}+\left((-1)^{\sigma\left(i_{k}\right)} Y h_{k}(X, Y) / a^{m}\right) \psi_{n+1} e_{\sigma\left(i_{k}\right)}{ }^{t} e_{1} \\
& +\left((-1)^{\sigma\left(i_{k}\right)} Y h_{k}(X, Y) / a^{m}\right) \psi_{n+1} e_{1}^{t} e_{\sigma\left(i_{k}\right)} \text {, for } i_{k} \geq 3 .
\end{aligned}
$$

Let $\varepsilon_{1}, \ldots, \varepsilon_{2 n}$ be the rows of the matrix $\left((1 \perp \varepsilon)^{t}\right)^{-1} \in \mathrm{E}_{2 n}\left(R_{a}, I_{a}\right)$. Let $\widetilde{e_{i}}$ denote the row vector $e_{i}\left(\left(I_{3} \perp \varepsilon\right)^{t}\right)^{-1}$ of length $2 n+2$. Note that $\widetilde{e_{1}}=e_{1}, \widetilde{e_{2}}=e_{2}$, and $\widetilde{e_{i}}=\left(0,0, \varepsilon_{i-2}\right)$, for $i \geq 3$. Using Lemma 5.16 we can write $\alpha\left(Y^{4^{r}} X\right)_{a}$ as product of elements of the form

$$
\begin{aligned}
& I_{2 n+2}+\left(Y h_{k}(X, Y) / a^{m}\right)\left(\begin{array}{cc}
\psi_{1} & 0 \\
0 & \varphi
\end{array}\right){\widetilde{e_{2}}}^{t} \widetilde{e_{2}}=\rho_{\varphi}\left(0, Y h_{k}(X, Y) / a^{m}\right), \\
& I_{2 n+2}+\left(Y h_{k}(X, Y) / a^{m}\right)\left(\begin{array}{cc}
\psi_{1} & 0 \\
0 & \varphi
\end{array}\right){\widetilde{e_{2}}}^{t} \widetilde{e_{j_{k}}}+\left(Y h_{k}(X, Y) / a^{m}\right)\left(\begin{array}{cc}
\psi_{1} & 0 \\
0 & \varphi
\end{array}\right) \widetilde{{e_{k}}^{t}}{\widetilde{e_{2}}}^{t} \\
& =\rho_{\varphi}\left(\left(Y h_{k}(X, Y) / a^{m}\right) \varepsilon_{j_{k}-2}, 0\right), \\
& I_{2 n+2}-\left(Y h_{k}(X, Y) / a^{m}\right)\left(\begin{array}{cc}
\psi_{1} & 0 \\
0 & \varphi
\end{array}\right){\widetilde{e_{1}}}^{t} \widetilde{e_{1}}=\mu_{\varphi}\left(0, Y h_{k}(X, Y) / a^{m}\right) \text {, } \\
& I_{2 n+2}-(-1)^{\sigma\left(i_{k}\right)}\left(Y h_{k}(X, Y) / a^{m}\right)\left(\begin{array}{cc}
\psi_{1} & 0 \\
0 & \varphi
\end{array}\right) \widetilde{e_{\sigma\left(i_{k}\right)}}{\widetilde{e_{1}}}^{t} \\
& -(-1)^{\sigma\left(i_{k}\right)}\left(Y h_{k}(X, Y) / a^{m}\right)\left(\begin{array}{cc}
\psi_{1} & 0 \\
0 & \varphi
\end{array}\right) \widetilde{e}_{1}^{t} \widetilde{e_{\sigma\left(i_{k}\right)}}=\mu_{\varphi}\left((-1)^{\sigma\left(i_{k}\right)}\left(Y h_{k}(X, Y) / a^{m}\right) \varepsilon_{\sigma\left(i_{k}\right)-2}, 0\right),
\end{aligned}
$$

for $i_{k}, j_{k} \geq 3$. Let $s \geq 0$ be an integer such that $\widetilde{\varepsilon_{i}}=a^{s} \varepsilon_{i} \in P$ for all $i=1, \ldots, 2 n$. Let $d^{\prime}=s+m$. Therefore $\alpha\left(\left(a^{d^{\prime}} Y\right)^{4^{r}} X\right)_{a}$ is product of elements of the form

$$
\begin{aligned}
& \rho_{\varphi}\left(0, a^{d^{\prime}} Y h_{k}\left(X, a^{d^{\prime}} Y\right) / a^{m}\right), \rho_{\varphi}\left(\left(a^{d^{\prime}} Y h_{k}\left(X, a^{d^{\prime}} Y\right) / a^{m}\right) \varepsilon_{j_{k}-2}, 0\right), \\
& \mu_{\varphi}\left(0, a^{d^{\prime}} Y h_{k}\left(X, a^{d^{\prime}} Y\right) / a^{m}\right) \text {, and } \mu_{\varphi}\left((-1)^{\sigma\left(i_{k}\right)}\left(a^{d^{\prime}} Y h_{k}\left(X, a^{d^{\prime}} Y\right) / a^{m}\right) \varepsilon_{\sigma\left(i_{k}\right)-2}\right) \text {. }
\end{aligned}
$$

Substituting $Y=1$ we get $\alpha\left(a^{d} X\right)_{a}$ is product of elements of the forms

$$
\begin{gathered}
\rho_{\varphi}\left(0, a^{s} h_{k}^{\prime}(X)\right), \rho_{\varphi}\left(a^{s} h_{k}^{\prime}(X) \varepsilon_{j_{k}-2}, 0\right), \\
\mu_{\varphi}\left(0, a^{s} h_{k}^{\prime}(X)\right), \text { and } \mu_{\varphi}\left((-1)^{\sigma\left(i_{k}\right)} a^{s} h_{k}^{\prime}(X) \varepsilon_{\sigma\left(i_{k}\right)-2}, 0\right) .
\end{gathered}
$$

Let us set $\alpha^{*}(X)$ to be the product of elements of the forms

$$
\begin{gathered}
\rho\left(0, a^{s} h_{k}^{\prime}(X)\right), \rho\left(h_{k}^{\prime}(X) \widetilde{\varepsilon_{j_{k}-2}}, 0\right), \\
\mu\left(0, a^{s} h_{k}^{\prime}(X)\right), \text { and } \mu\left((-1)^{\sigma\left(i_{k}\right)} h_{k}^{\prime}(X) \widetilde{\varepsilon_{\sigma\left(i_{k}\right)-2}}, 0\right) .
\end{gathered}
$$

Note that $\alpha^{*}(X)$ belongs to $\operatorname{ETranssp}_{S}(Q[X],\langle\rangle,) \cap \operatorname{Aut}(Q[X], I Q[X])$. Generators of ETrans $\operatorname{Sp}_{\mathrm{p}}(Q[X])$ have the splitting property as

$$
\begin{aligned}
\rho\left(q_{1} / 2, \alpha_{1} / 2\right) \rho\left(q_{2}, \alpha_{2}\right) \rho\left(q_{1} / 2, \alpha_{1} / 2\right) & =\rho\left(q_{1}+q_{2}, \alpha_{1}+\alpha_{2}\right), \\
\mu\left(q_{1} / 2, \alpha_{1} / 2\right) \mu\left(q_{2}, \alpha_{2}\right) \mu\left(q_{1} / 2, \alpha_{1} / 2\right) & =\mu\left(q_{1}+q_{2}, \alpha_{1}+\alpha_{2}\right) .
\end{aligned}
$$


Therefore, using argument similar to Lemma 2.10 we can show that $\alpha^{*}(X)$ is in ETrans $_{\mathrm{Sp}}(Q[X], I Q[X],\langle\rangle$,$) . It is clear from construction that \alpha^{*}(0)=I d$. and $\alpha^{*}(X)$ localises to $\alpha(b X)$, for some $b \in\left(a^{d}\right), d \gg 0$.

Lemma 5.20. Let $R$ be a commutative ring with $R=2 R$, and let $I$ be an ideal of $R$. Let $(P,\langle\rangle$,$) be a symplectic R$-module with $P$ finitely generated projective module of rank $2 n, n \geq 1$. Let $\alpha(X) \in \operatorname{Sp}(Q[X],\langle\rangle$,$) , with \alpha(0)=I d$. If for each maximal ideal $\mathfrak{m}$ of $R, \alpha(X)_{\mathfrak{m}} \in \operatorname{ETrans}_{\operatorname{Sp}}\left(Q_{\mathfrak{m}}[X], I Q_{\mathfrak{m}}[X],\langle,\rangle_{\psi_{1} \perp \varphi_{\mathfrak{m}}}\right)$, where $\varphi_{\mathfrak{m}} \equiv \psi_{n}(\bmod I)$, then $\alpha(X) \in$ ETranssp $\left._{\text {Th }}(X], I Q[X],\langle\rangle,\right)$.

Proof: Proof follows arguing similarly as in Lemma 4.7.

Theorem 5.21. Let $R$ be a commutative ring with $R=2 R$, and let $I$ be an ideal of $R$. Let $(P,\langle\rangle$,$) be a symplectic R$-module with $P$ a finitely generated projective module of rank $2 n, n \geq 1$. Let $q(X)=(a(X), b(X), p(X)) \in \operatorname{Um}(Q[X], I Q[X])$. If for each maximal ideal $\mathfrak{m}$ of $R$, we have $q(X) \in q(0) \operatorname{ETrans}_{\operatorname{Sp}}\left(Q_{\mathfrak{m}}[X], I Q_{\mathfrak{m}}[X],\langle,\rangle_{\psi_{1} \perp \varphi_{\mathfrak{m}}}\right)$, where $\varphi_{\mathfrak{m}} \equiv \psi_{n}(\bmod I)$, then $q(X) \in q(0) \operatorname{ETrans}_{\mathrm{Sp}}(Q[X], I Q[X],\langle\rangle$,$) .$

Proof: Proof follows arguing similarly as in Theorem 4.8 .

We now establish equality of the symplectic transvection group and the elementary symplectic transvection group. Before that we prove a lemma to show that symplectic transvections are homotopic to identity.

Lemma 5.22. Let $(P,\langle\rangle$,$) be a symplectic R$-module and $\alpha \in \operatorname{Transsp}_{\mathrm{S}}(P,\langle\rangle$,$) . Then$ there exists $\beta(X) \in \operatorname{Trans}_{\mathrm{Sp}}(P[X],\langle\rangle$,$) such that \beta(1)=\alpha$ and $\beta(0)=I d$.

Proof: As $\alpha \in \operatorname{Trans}_{\mathrm{Sp}}(P,\langle\rangle$,$) , it is product of symplectic transvections of the$ form $\sigma$, where $\sigma$ takes $p \in P$ to $p+\langle u, p\rangle v+\langle v, p\rangle u+a\langle u, p\rangle u$, where $a \in R, u, v \in P$ are fixed elements with $\langle u, v\rangle=0$, and either $u$ or $v$ is unimodular. Define $\sigma X$ as the map which takes $p \in P$ to either $p+\langle u, p\rangle v X+\langle v X, p\rangle u+a X\langle u, p\rangle u$ or $p+\langle u X, p\rangle v+\langle v, p\rangle u X+a\langle u X, p\rangle u X$. This choice depends on whether $u$ is unimodular or $v$ is unimodular. Note that $u X$ represents $u$ times $X, v X$ represents $v$ times $X$, and $a X$ represents a times $X$. Also, note that $u X, v X \in P[X]$ and $\alpha X \in R[X]$. We set $\beta(X)$ to be the product of elements of the form $\sigma X$, whenever $\sigma$ appears in the expression of $\alpha$. Then $\beta(1)=\alpha$ and $\beta(0)=I d$.

Theorem 5.23. Let $R$ be a commutative ring with $R=2 R$, and let $I$ be an ideal of $R$. Let $(P,\langle\rangle$,$) be a symplectic R$-module with $P$ a finitely generated projective module of rank $2 n, n \geq 1$. Also assume that over the local ring $R_{\mathfrak{m}}$ for any maximal ideal $\mathfrak{m}$ of $R$, the alternating form $\langle$,$\rangle corresponds to the alternating matrix \varphi_{\mathfrak{m}}$ (w.r.t. some basis $)$, where $\varphi_{\mathfrak{m}} \equiv \psi_{n}(\bmod I)$. Then $\operatorname{Trans}_{\mathrm{Sp}}(Q, I Q,\langle\rangle)=,\operatorname{ETrans}_{\mathrm{Sp}}(Q, I Q,\langle\rangle$,$) .$

Proof: We have ETrans $\operatorname{Trp}_{\mathrm{Sp}}(Q, I Q,\langle\rangle,) \subseteq \operatorname{Trans}_{\mathrm{Sp}}(Q, I Q,\langle\rangle$,$) . We need to show$ other inclusion. Let us choose $\alpha$ from $\operatorname{Trans}_{\mathrm{sp}}(Q, I Q,\langle\rangle$,$) . By Lemma 5.22$ there exists $\alpha(X)$ in $\operatorname{Transsp}_{\mathrm{Sp}}(Q[X], I Q[X],\langle\rangle$,$) such that \alpha(1)=\alpha$ and $\alpha(0)=I d$. Note that $\operatorname{Transsp}_{\mathrm{Sp}}\left(Q_{\mathfrak{m}}[X], I Q_{\mathfrak{m}}[X],\langle,\rangle_{\psi_{1} \perp \varphi_{\mathfrak{m}}}\right)=\operatorname{ETranssp}_{\operatorname{Tr}_{\mathfrak{m}}}\left(Q_{\mathfrak{m}}[X], I Q_{\mathfrak{m}}[X],\langle,\rangle_{\psi_{1} \perp \varphi_{\mathfrak{m}}}\right)$, for each maximal ideal $\mathfrak{m}$ of $R$ (follows from Remark [5.18). Hence $\alpha(X)_{\mathfrak{m}}$ belongs to $\operatorname{ETrans}_{\mathrm{Sp}}\left(Q_{\mathfrak{m}}[X], I Q_{\mathfrak{m}}[X],\langle,\rangle_{\psi_{1} \perp \varphi \otimes R_{\mathfrak{m}}[X]}\right)$, for each maximal ideal $\mathfrak{m}$ of $R$. Therefore, $\alpha(X) \in$ ETranssp $_{\text {ST }}(Q[X], I Q[X],\langle\rangle$,$) (see Lemma [5.20). Substituting X=1$ we get the result. 


\section{Equality of orbits}

In this section we establish main result of this article regarding equality of orbits.

Theorem 6.1. Let $R$ be a commutative ring with $R=2 R$, and let $I$ be an ideal of $R$. Let $(P,\langle\rangle$,$) be a symplectic R$-module with $P$ finitely generated projective module of rank $2 n, n \geq 1$. Let $Q=R^{2} \oplus P$ and $v=(a, b, p) \in \operatorname{Um}(Q, I Q)$. We also assume for each maximal ideal $\mathfrak{m}$ of $R$, the alternating form $\langle$,$\rangle over the local ring R_{\mathfrak{m}}$ corresponds to an alternating matrix $\varphi_{\mathfrak{m}}$ (w.r.t. some basis), such that $\varphi_{\mathfrak{m}}=(1 \perp \varepsilon(\mathfrak{m}))^{t} \psi_{n}(1 \perp$ $\varepsilon(\mathfrak{m}))$, for some $\varepsilon(\mathfrak{m}) \in E_{2 n-1}\left(R_{\mathfrak{m}}, I_{\mathfrak{m}}\right)$. Then

$$
(a, b, p) \operatorname{ETrans}(Q, I Q)=(a, b, p) \operatorname{ETrans}_{\mathrm{Sp}}(Q, I Q,\langle,\rangle) .
$$

Proof: Let $\alpha \in \operatorname{ETrans}(Q, I Q)$. Let us choose $\alpha(X)$ from $\operatorname{ETrans}(Q[X], I Q[X])$ such that $\alpha(1)=\alpha$ and $\alpha(0)=I d$ (see Lemma 4.9). Let us define $V(X)=$ $(a, b, p) \alpha(X)$.

Let $\mathfrak{m}$ be a maximal ideal of $R$. Over $R_{\mathfrak{m}}$, we have $\varphi_{\mathfrak{m}}=(1 \perp \varepsilon(\mathfrak{m}))^{t} \psi_{n}(1 \perp \varepsilon(\mathfrak{m}))$, where $\varepsilon(\mathfrak{m}) \in \mathrm{E}_{2 n}\left(R_{\mathfrak{m}}, I_{\mathfrak{m}}\right)$. Let us define $W(X)=V(X)(1 \perp \varepsilon(\mathfrak{m}))^{t}$. We have

$$
\begin{aligned}
W(X) & \in W(0) \mathrm{E}_{2 n+2}\left(R_{\mathfrak{m}}[X], I_{\mathfrak{m}}[X]\right) \\
& =W(0) \operatorname{ESp}_{2 n+2}\left(R_{\mathfrak{m}}[X], I_{\mathfrak{m}}[X]\right) \\
& \left.=W(0) \operatorname{ETrans}_{\mathfrak{s p}}\left(Q_{\mathfrak{m}}[X], I Q_{\mathfrak{m}}[X]\right),\langle,\rangle_{\psi_{n+1}}\right)
\end{aligned}
$$

and hence $V(X) \in V(0) \operatorname{ETranssp}_{\mathrm{T}_{\mathrm{p}}}\left(Q_{\mathfrak{m}}[X], I Q_{\mathfrak{m}}[X],\langle,\rangle_{\psi_{1} \perp \varphi_{\mathfrak{m}}}\right)$. The first equality above follows from ([7], Theorem 5.6) when $n>2$. For $n=2$ the result has been proved in the Appendix (see Theorem 7.11). This is true for all maximal ideal $\mathfrak{m}$ of $R$, hence by Theorem 5.21, $V(X) \in V(0)$ ETranssp $\left._{\text {Th }}(X], I Q[X],\langle\rangle,\right)$. Substituting $X=1$ we get $(a, b, p) \alpha \in(a, b, p) \operatorname{ETranssp}_{\mathrm{Sp}}(Q, I Q,\langle\rangle$,$) .$

Now we show the other inclusion. Let $\beta \in \operatorname{ETranssp}_{\text {Th }}(Q, I Q,\langle\rangle$,$) . Let us choose$ $\beta(X)$ from ETranssp $(Q[X], I Q[X],\langle\rangle$,$) such that \beta(1)=\beta$ and $\beta(0)=I d$ (see Lemma 5.22). We define $V(X)=(a, b, p) \beta(X)$.

Let $R_{\mathfrak{m}}$ be the local ring at the maximal ideal $\mathfrak{m}$. We define $W(X)=V(X)(1 \perp$ $\varepsilon(\mathfrak{m}))^{t}$. We have

$$
\begin{aligned}
& W(X) \in W(0)\left((1 \perp \varepsilon(\mathfrak{m}))^{t}\right)^{-1} \operatorname{ETrans}_{\mathrm{Sp}}\left(Q_{\mathfrak{m}}[X], I Q_{\mathfrak{m}}[X],\langle,\rangle_{\psi_{1} \perp \varphi_{\mathfrak{m}}}\right)(1 \perp \varepsilon(\mathfrak{m}))^{t} \\
& =W(0) \operatorname{ETrans}_{\mathrm{Sp}}\left(Q_{\mathfrak{m}}[X], I Q_{\mathfrak{m}}[X],\langle,\rangle_{\psi_{n+1}}\right) \\
& =W(0) \operatorname{ESp}_{2 n+2}\left(R_{\mathfrak{m}}[X], I_{\mathfrak{m}}[X]\right) \\
& =W(0) \mathrm{E}_{2 n+2}\left(R_{\mathfrak{m}}[X], I_{\mathfrak{m}}[X]\right) \text {, }
\end{aligned}
$$

and hence $V(X) \in V(0) \operatorname{ETrans}\left(Q_{\mathfrak{m}}[X], I Q_{\mathfrak{m}}[X]\right)$. The last equality above follows from ([7], Theorem 5.6) when $n>2$. For $n=2$ the result has been proved in the Appendix (see Theorem 7.11). This is true for all maximal ideal $\mathfrak{m}$ of $R$, hence by Theorem $4.8, V(X) \in V(0)$ ETrans $(Q[X], I Q[X])$. Substituting $X=1$, we get $(a, b, p) \beta \in(a, b, p) \operatorname{ETrans}(Q, I Q)$. 


\section{Decrease in injective stability for $\operatorname{Sp}(Q,\langle\rangle,) / \operatorname{ETrans}_{\mathrm{Sp}}(Q,\langle\rangle$,}

Final goal of this section is to give an improvement for Basu-Rao ([6], Theorem 2) estimate in the module case over finitely generated rings. For this purpose we state and prove a few preliminary results. While dealing with the results in the relative case w.r.t. an ideal I of a ring $R$, we will always assume that over the local ring $R_{\mathfrak{m}}$, where $\mathfrak{m}$ is a maximal ideal, the alternating form $\langle$,$\rangle corresponds to the alternating matrix$ $\varphi_{\mathfrak{m}}$ (w.r.t. some basis), where $\varphi_{\mathfrak{m}} \equiv \psi_{n}(\bmod I)$.

Theorem 7.1. (4), Theorem 3.4, Page 183) Let $R$ be a commutative ring of dim $d$. Let $I$ be an ideal of $R$ and $P$ be a projective module of rank $\geq d+1$. Let $\tilde{Q}=R \oplus P$. Let $v_{1}, v_{2} \in \operatorname{Um}(\tilde{Q})$ with $v_{1} \equiv v_{2}(\bmod I \tilde{Q})$. Then there exists $\beta \in \operatorname{ETrans}(\tilde{Q}, I \tilde{Q})$ such that $v_{1} \beta=v_{2}$.

Theorem 7.2. ([16, Theorem 17.2$)$ Let $R$ be a finitely generated $\mathbb{Z}$-algebra and $k R=R$ for some natural number $k \geq 2$. Then $\operatorname{sr}(R) \leq \max (2, \operatorname{dim}(R))$.

Theorem 7.3. ([16], Corollary 17.3) Let $R$ be a finitely generated algebra over a field which is algebraic over a finite field. Then $\operatorname{sr}(R) \leq \max (2, \operatorname{dim}(R))$.

Using the above three results one can prove the following theorem.

Theorem 7.4. ([12], Theorem 2.4) Let $R$ be a finitely generated ring over $\mathbb{Z}$, or over a field $K$ which is either finite or algebraic closure of a finite field. Let the dimension of $R$ be $d(d \geq 2)$ and $I$ be an ideal of $R$. Let $P$ be a projective module of rank $\geq d$ and $\tilde{Q}=R \oplus P$. Let $(a, x) \in R \oplus P$ be a unimodular element such that $(a, x) \equiv$ $(1,0)(\bmod I \tilde{Q})$. Then there exists $\varepsilon \in \operatorname{ETrans}(\tilde{Q}, I \tilde{Q})$ such that $(a, x) \varepsilon=(1,0)$.

Lemma 7.5. Let $R$ be a finitely generated ring over $\mathbb{Z}$, or over a field $K$ which is either finite or algebraic closure of a finite field. Let the dimension of $R$ be $d$, and $I$ be an ideal of $R$. Let us assume $R=2 R$. Let $(P,\langle\rangle$,$) be a symplectic R$-module with $P$ finitely generated projective module of even rank $\geq \max \{2, d-1\}$, and let $Q=R^{2} \oplus P$. Let $v_{1}, v_{2} \in \operatorname{Um}(Q)$ with $v_{2}=(1,0)$ and $v_{1} \equiv v_{2}(\bmod I Q)$. Then there exists $\beta \in \operatorname{ETrans}_{\mathrm{Sp}}(Q, I Q)$ such that $v_{1} \beta=v_{2}$.

Proof: Follows from Theorem 7.4 and Theorem 6.1.

Theorem 7.6. Let $R$ be a finitely generated ring over $\mathbb{Z}$, or over a field $K$ which is either finite or algebraic closure of a finite field. Let the dimension of $R$ be $d$. Let us assume $R=2 R$. Let $(P,\langle\rangle$,$) be a symplectic R$-module with $P$ finitely generated projective module of even rank $\geq d-3$. Let $Q=\left(R^{2} \oplus P\right)$, and let $\widehat{Q}=\left(R^{2} \oplus Q\right)$. Let $\sigma \in \operatorname{Sp}(Q,\langle\rangle$,$) with \left(I_{2} \perp \sigma\right) \in \operatorname{ETranssp}_{\mathrm{S}}(\widehat{Q},\langle\rangle$,$) . Then \sigma$ is (stably elementary symplectic) homotopic to the identity. In fact, $\sigma=\rho(1)$, with $\rho(0)=I d$., for some $\rho(X) \in \operatorname{Sp}(Q[X],\langle\rangle$,$) such that \left(I_{2} \perp \rho(X)\right) \in \operatorname{ETranssp}_{\operatorname{Si}}(\widehat{Q}[X],\langle\rangle$,$) .$

Proof: Let us choose $\alpha(X)$ from ETrans $\operatorname{Sp}_{\mathrm{p}}(\widehat{Q}[X],\langle\rangle$,$) , such that \alpha(1)=\left(I_{2} \perp \sigma\right)$, and $\alpha(0)=I d$. (see Lemma 5.22). Let $v(X)=e_{1} \alpha(X)$. Note that $v(X)$ belongs to $\operatorname{Um}\left(\widehat{Q}[X],\left(X^{2}-X\right) \widehat{Q}[X]\right)$. Also, $e_{1}=(1,0,0)$ belongs to $\operatorname{Um}\left(\widehat{Q}[X],\left(X^{2}-X\right) \widehat{Q}[X]\right)$. 
Therefore, by Lemma 7.5, we have $\beta(X) \in \operatorname{ETrans}_{\mathrm{Sp}}\left(\widehat{Q}[X],\left(X^{2}-X\right) \widehat{Q}[X],\langle\rangle,\right)$, such that $v(X) \beta(X)=(1,0,0)$, i.e, $e_{1} \alpha(X) \beta(X)=e_{1}$. Let $\delta(X)$ denote the product $\alpha(X) \beta(X)$. Note that $\delta(X) \in$ ETranssp $_{\mathrm{S}}(\widehat{Q}[X],\langle\rangle$,$) and e_{1} \delta(X)=e_{1}$. Therefore, $\delta(X)$ will be of the form

$$
\left(\begin{array}{ccc}
1 & 0 & 0 \\
* & 1 & * \\
* & 0 & \gamma(X)
\end{array}\right),
$$

where $\gamma(X) \in \operatorname{Sp}(Q[X],\langle\rangle$,$) . Note that \delta(1)=\left(I_{2} \perp \sigma\right)$. Therefore $\gamma(1)=\rho$, $\gamma(0)=I d$., and hence $\rho$ is (symplectic) homotopic to identity.

Theorem 7.7. ([6], Theorem 3.13) Let $(R, \mathfrak{m})$ be a regular local ring. Assume that $R$ contains a field, or characteristic of $R / \mathfrak{m}$ is not in $\mathfrak{m}^{2}$. Then

$$
\operatorname{Sp}\left(R[X]^{2 n+2},\langle,\rangle_{\varphi_{\mathfrak{m}}}\right)=\operatorname{ETrans}_{\mathrm{Sp}}\left(R[X]^{2 n+2},\langle,\rangle_{\varphi_{\mathfrak{m}}}\right),
$$

for $n \geq 1$, where $\varphi_{\mathfrak{m}}$ is the associated matrix of the alternating bilinear form $\langle$,$\rangle (w.r.t.$ some basis).

The next corollary improves Basu-Rao estimate in the module case over finitely generated rings.

Corollary 7.8. Let $R$ be a finitely generated non-singular algebra of dimension $d$ over $K$, where $K$ is either $\mathbb{Z}$, or a finite field or the algebraic closure of a finite field. Let us assume $R=2 R$. Let $(P,\langle\rangle$,$) be a symplectic R$-module with $P$ finitely generated projective module of even rank $\geq \max \{2, d-3\}$. Let $Q=\left(R^{2} \oplus P\right)$, and $\widehat{Q}=\left(R^{2} \oplus Q\right)$. If $\sigma \in \operatorname{Sp}(Q,\langle\rangle$,$) with \left(I_{2} \perp \sigma\right) \in \operatorname{ETrans}_{\mathrm{Sp}}(\widehat{Q},\langle\rangle$,$) , then \sigma$ belongs to $\operatorname{ETrans}_{\mathrm{Sp}}(Q,\langle\rangle$,$) .$

Proof: From the proof of Theorem 7.6 it follows that $\sigma=\rho(1)$ for some $\rho(X) \in$ $\operatorname{Sp}(Q[X],\langle\rangle$,$) , with \rho(0)=I d$. Using Theorem 7.7 we get that $\operatorname{Sp}\left(R_{\mathfrak{m}}[X]^{2 n+2},\langle,\rangle_{\varphi_{\mathfrak{m}}}\right)$ $=\operatorname{ETrans}_{S_{p}}\left(R_{\mathfrak{m}}[X]^{2 n+2},\langle,\rangle_{\varphi_{\mathfrak{m}}}\right)$, for all maximal ideals $\mathfrak{m}$ of $R$. This implies $\rho(X) \in$ ETranssp $_{S}\left(R_{\mathfrak{m}}[X]^{2 n+2},\langle,\rangle_{\varphi_{\mathfrak{m}}}\right)$, for all maximal ideals $\mathfrak{m}$ in $R$. Therefore, $\rho(X) \in$ $\operatorname{ETranssp}_{\mathrm{Sp}}(R[X],\langle\rangle$,$) (see Lemma 5.20). Hence \sigma=\rho(1)$ belongs to $\operatorname{ETranssp}_{\mathrm{S}}(R,\langle\rangle$,$) .$

\section{Appendix}

\section{Alpesh Dhorajia}

Birla Institute of Technology and Science, K. K. Birla Goa Campus, Goa 403 726, India

The aim of this Appendix is to work out via commutator laws the size 4 cases in the study above. This entails proving Lemma 2.10 and Lemma 3.1 above in the symplectic size 4 case.

In order to prove Lemma 2.10 in the symplectic case we need to establish the following inclusion first. 
Lemma 7.9. Let $n \geq 2, R$ be a commutative ring with $R=2 R$, and let $I$ be an ideal of $R$. Then $\operatorname{ESp}_{2 n}(R, I) \subseteq \operatorname{ESp}_{2 n}^{1}(R, I)$.

Proof: It suffices to show $\operatorname{ESp}_{2 n}^{1}(R, I)$ contains the set $S_{i j}=\left\{s e_{i j}(a) s e_{j i}(x) s e_{i j}(-a)\right.$ : $a \in R, x \in I\}$, for all $i, j$, with $i \neq j$. Note that $S_{i j}=S_{\sigma(j) \sigma(i)}$ when $i \neq \sigma(j)$. First we state the following identities

$$
\begin{aligned}
& {[g h, k]=\left({ }^{g}[h, k]\right)[g, k],} \\
& {[g, h k]=[g, h]\left({ }^{h}[g, k]\right),} \\
& { }^{g}[h, k]=\left[{ }^{g} h,{ }^{g} k\right],
\end{aligned}
$$

where ${ }^{g} h$ denotes $g h g^{-1}$ and $[g, h]=g h g^{-1} h^{-1}$. These identities will be used throughout the proof without mentioning it always.

Also, we need the following relations which hold for all integers $i, j$ with $i \neq j, \sigma(j)$, and for all $a, b \in R$.

$$
\begin{array}{r}
{\left[s e_{i \sigma(i)}(a), s e_{\sigma(i) j}(b)\right]=s e_{i j}(a b) s e_{\sigma(j) j}\left((-1)^{i+j} a b^{2}\right),} \\
{\left[s e_{i k}(a), s e_{k j}(b)\right]=s e_{i j}(a b), \text { if } k \neq \sigma(i), \sigma(j),} \\
{\left[s e_{i k}(a), s e_{k \sigma(i)}(b)\right]=s e_{i \sigma(i)}(2 a b), \text { if } k \neq i, \sigma(i) .}
\end{array}
$$

The following relation holds for all $i, j$ with $1 \leq i \neq j \leq 2 n$, and for all $a, b \in R$.

$$
\left[s e_{i j}(a), s e_{k l}(b)\right]=I d \text {., if } i \neq l, \sigma(k) \text { and } j \neq k, \sigma(l) .
$$

Note that $s e_{1 j}(x), s e_{i 1}(x) \in \operatorname{ESp}_{2 n}^{1}(R, I)$, for $2 \leq i, j \leq 2 n$ and $x \in I$. For $3 \leq$ $i, j \leq 2 n$ with $i \neq \sigma(j)$, and $x \in I$, we have $s e_{i j}(x)=\left[\operatorname{se}_{i 1}(x), s_{1 j}(1)\right] \in \operatorname{ESp}_{2 n}^{1}(R, I)$ and $s e_{i \sigma(i)}(x)=\left[s e_{i 1}(x / 2), s e_{1 \sigma(i)}(1)\right] \in \operatorname{ESp}_{2 n}^{1}(R, I)$. In the following computation we will express the generators of $S_{i j}$ in terms of $s e_{i j}(x)$ or $s e_{1 j}(a)$, where $x \in I, a \in R$. Also, note that we will use $*$ to represent elements of the ideal $I$ and $\circledast$ to represent elements of the ring $R$, which does not belong to the ideal $I$.

Case (a): It is clear that $S_{1 j} \subseteq \operatorname{ESp}_{2 n}^{1}(R, I)$, for $2 \leq j \leq 2 n$.

Case (b): Here we show $S_{i 1} \subseteq \operatorname{ESp}_{2 n}^{1}(R, I)$, for $3 \leq i \leq 2 n$. Let $a \in R$ and $x \in I$. We have

$$
\begin{aligned}
& s e_{i j}(a) s e_{j i}(x) \\
& =s e_{i j}(a)\left(\left[s e_{j \sigma(j)}(1), s e_{\sigma(j) i}(*)\right] s e_{\sigma(i) i}(*)\right) \\
& =\left[s e_{j \sigma(i)}(\circledast) s e_{i \sigma(i)}(\circledast) s e_{j \sigma(j)}(1), s e_{\sigma(j) j}(*) s e_{\sigma(j) i}(*)\right]^{s e_{i j}(a)} s e_{\sigma(i) i}(*) \\
& =\left[s e_{i \sigma(i)}(\circledast) s e_{j \sigma(i)}(\circledast) s e_{j \sigma(j)}(1), s e_{\sigma(j) j}(*) s e_{\sigma(j) i}(*)\right]^{s e_{i j}(a)} s e_{\sigma(i) i}(*) \\
& =\left({ }^{s e_{i \sigma(i)}(\circledast)}\left[s e_{j \sigma(i)}(\circledast) s e_{j \sigma(j)}(1), s e_{\sigma(j) j}(*) s e_{\sigma(j) i}(*)\right]\right) \\
& {\left[s e_{i \sigma(i)}(\circledast), s e_{\sigma(j) j}(*) s e_{\sigma(j) i}(*)\right]^{s e_{i j}(a)} s e_{\sigma(i) i}(*)} \\
& =A B C \text { (say), }
\end{aligned}
$$


where

$$
\begin{array}{rl}
A & =s e_{i \sigma(i)}(\circledast) \\
B & =\left[s e_{j \sigma(i)}(\circledast) s e_{j \sigma(j)}(1), s e_{\sigma(j) j}(*) s e_{\sigma(j) i}(*)\right], \\
C & =s e_{i j}(a) \\
B & s e_{\sigma(i) i}(*) .
\end{array}
$$

Now

$$
\begin{aligned}
& A=s e_{i \sigma(i)}(\circledast)\left[s e_{j \sigma(i)}(\circledast) s e_{j \sigma(j)}(1), s e_{\sigma(j) j}(*) s e_{\sigma(j) i}(*)\right], \\
& =\left[s e_{j \sigma(i)}(\circledast) s e_{j \sigma(j)}(1), s e_{\sigma(j) j}(*) s e_{i j}(*) s e_{\sigma(j) j}(*) s e_{\sigma(j) i}(*)\right] \\
& =\left[s e_{j \sigma(j)}(1) s e_{j \sigma(i)}(\circledast), s e_{\sigma(j) j}(*) s e_{i j}(*) s e_{\sigma(j) i}(*)\right] \text {, } \\
& B=\left[s e_{i \sigma(i)}(\circledast), s e_{\sigma(j) j}(*) s e_{\sigma(j) i}(*)\right] \\
& =s e_{i \sigma(i)}(\circledast) s e_{\sigma(j) j}(*)^{s e_{i \sigma(i)}(\circledast)} s e_{\sigma(j) i}(*) s e_{\sigma(j) i}(*)^{-1} s e_{\sigma(j) j}(*)^{-1} \\
& =s e_{\sigma(j) j}(*) s e_{i j}(*) s e_{\sigma(j) j}(*) s e_{\sigma(j) i}(*) s e_{\sigma(j) i}(*) s e_{\sigma(j) j}(*), \\
& \left.C=\operatorname{se}_{i j}(a) s e_{\sigma(i) i}(*)\right) \\
& \left.=s e_{\sigma(i) j}(*) s e_{\sigma(j) j}(*) s e_{\sigma(i) i}(*)\right) .
\end{aligned}
$$

It is clear that $B, C \in \operatorname{ESp}_{2 n}^{1}(R, I)$. In this case we have $j=1$, and hence $A \in \operatorname{ESp}_{2 n}^{1}(R, I)$.

Case (c): Here we show $S_{i j} \subseteq \operatorname{ESp}_{2 n}^{1}(R, I)$, for $3 \leq i, j \leq 2 n$, and $j=\sigma(i)$. Note that

$$
\begin{aligned}
& s e_{i \sigma(i)}(a) \\
& s e_{\sigma(i) i}(x) \\
&= s e_{i \sigma(i)}(a)\left[s e_{\sigma(i) 1}(x / 2), s e_{1 i}(1)\right] \\
&= {\left[s e_{i 1}(*) s e_{21}(*) s e_{\sigma(i) 1}(*), s e_{i 2}(\circledast) s e_{12}(\circledast) s e_{1 i}(1)\right] } \\
&= {\left[s e_{i 1}(*) s e_{21}(*) s e_{\sigma(i) 1}(*), s e_{1 \sigma(i)}(\circledast) s e_{12}(\circledast) s e_{1 i}(1)\right] } \\
& \in \operatorname{ESp}_{2 n}^{1}(R, I) .
\end{aligned}
$$

Case (d): Here we show $S_{i j} \subseteq \operatorname{ESp}_{2 n}^{1}(R, I)$, for $3 \leq i, j \leq 2 n$, and $j \neq \sigma(i)$. From the computaion in Case (b) it is clear that ${ }^{s e_{i j}(a)} s_{j i}(x)$ can be expressed as product of $A B C$, where $B, C \in \operatorname{ESp}_{2 n}^{1}(R, I)$. Note that

$$
\begin{aligned}
& A=\left[s e_{j \sigma(j)}(1) s e_{j \sigma(i)}(\circledast), s e_{\sigma(j) j}(*) s e_{i j}(*) s e_{\sigma(j) i}(*)\right] \\
& =s e_{j \sigma(j)}(1) s e_{j \sigma(i)}(\circledast) s e_{\sigma(j) j}(*) s e_{j \sigma(j)}(1) s e_{j \sigma(i)}(\circledast) s e_{i j}(*) s e_{j \sigma(j)}(1) s e_{j \sigma(i)}(\circledast) s e_{\sigma(j) i}(*) \\
& s e_{\sigma(j) i}(*)^{-1} s e_{i j}(*)^{-1} s e_{\sigma(j) j}(*)^{-1} \\
& =P Q R s e_{\sigma(j) i}(*) s e_{i j}(*) s e_{\sigma(j) j}(*),
\end{aligned}
$$

where

$$
\begin{aligned}
& P=s e_{j \sigma(j)}(1) s e_{j \sigma(i)}(\circledast) s e_{\sigma(j) j}(*), \\
& Q=s e_{j \sigma(j)}(1) s e_{j \sigma(i)}(\circledast) s e_{i j}(*), \\
& R=s e_{j \sigma(j)}(1) s e_{j \sigma(i)}(\circledast) s e_{\sigma(j) i}(*) \text {. }
\end{aligned}
$$


Now

$$
\begin{aligned}
& P=s e_{j \sigma(j)}(1) s e_{j \sigma(i)}(\circledast) s e_{\sigma(j) j}(*) \\
& =s e_{j \sigma(j)}(1)\left(s e_{\sigma(j) \sigma(i)}(*) s e_{i \sigma(i)}(*) s e_{\sigma(j) j}(*)\right) \\
& =s e_{j \sigma(i)}(*) s e_{i \sigma(i)}(*) s e_{\sigma(j) \sigma(i)}(*) s e_{i \sigma(i)}(*)\left({ }^{s e_{j \sigma(j)}(1)} s e_{\sigma(j) j}(*)\right) \text {, } \\
& Q=s e_{j \sigma(j)}(1) s e_{j \sigma(i)}(\circledast) s e_{i j}(*) \\
& =\operatorname{se}_{j \sigma(j)}{ }^{(1)} s e_{i \sigma(i)}(*) \\
& =\operatorname{se}_{i \sigma(i)}(*), \\
& R=s e_{j \sigma(j)}(1) s e_{j \sigma(i)}(\circledast) s e_{\sigma(j) i}(*) \\
& =s e_{j \sigma(i)}(\circledast) s e_{j \sigma(j)}(1) s e_{\sigma(j) i}(*) \\
& =s e_{j \sigma(i)}(\circledast)\left(s e_{j i}(*) s e_{\sigma(i) i}(*) s e_{\sigma(j) i}(*)\right) \\
& =s e_{j \sigma(i)}(\circledast)\left(s e_{j i}(*) s e_{\sigma(i) i}(*) s e_{\sigma(i) j}(*)\right) \\
& =s e_{j \sigma(j)}(*) s e_{j i}(*) s e_{\sigma(i) \sigma(j)}(*) s e_{j \sigma(j)}(*) s e_{\sigma(i) i}(*) \\
& s e_{j \sigma(i)}(\circledast) s e_{\sigma(i) j}(*) \\
& =s e_{j \sigma(j)}(*) s e_{j i}(*) s e_{j i}(*) s e_{j \sigma(j)}(*) s e_{\sigma(i) i}(*) \\
& s e_{j \sigma(i)}(\circledast)\left[s e_{\sigma(i) 1}(*), s e_{1 j}(1)\right] \\
& =s e_{j \sigma(j)}(*) s e_{j i}(*) s e_{j \sigma(j)}(*) s e_{\sigma(i) i}(*) \\
& {\left[s e_{j 1}(*) s e_{\sigma(i) 1}(*), s e_{1 \sigma(i)}(\circledast) s e_{1 j}(1)\right] .}
\end{aligned}
$$

and hence $A \in \operatorname{ESp}_{2 n}^{1}(R, I)$.

Case (e): Finally we show $S_{21} \subseteq \operatorname{ESp}_{2 n}^{1}(R, I)$. Note that

$$
\begin{aligned}
& { }^{s e_{21}(a)} s e_{12}(x) \\
= & { }^{s} e_{21}(a)\left[s e_{1 k}(1), s e_{k 2}(*)\right], \text { where } k \neq 1,2 \\
= & {\left[{ }^{s e_{21}(a)} s e_{1 k}(1), s e_{2 \sigma(k)}(*) s e_{k \sigma(k)}(*) s e_{k 2}(*)\right] } \\
= & {\left[\alpha, s e_{2 \sigma(k)}(*) s e_{k \sigma(k)}(*) s e_{k 2}(*)\right], \text { where } \alpha={ }^{s e_{21}(a)} s e_{1 k}(1) } \\
= & { }^{\alpha} s e_{2 \sigma(k)}(*)^{\alpha} s e_{k \sigma(k)}(*)^{\alpha} s e_{k 2}(*) s e_{k 2}(*)^{-1} s e_{k \sigma(k)}(*)^{-1} s e_{2 \sigma(k)}(*)^{-1} .
\end{aligned}
$$

The $\alpha$ above can be expressed in the following three different ways, namely

$$
\begin{aligned}
\alpha & =s e_{1 k}(1) s e_{2 k}(\circledast) s e_{\sigma(k) k}(\circledast) \\
& =s e_{2 k}(\circledast) s e_{\sigma(k) k}(\circledast) s e_{1 k}(1) \\
& =s e_{\sigma(k) k}(\circledast) s e_{2 k}(\circledast) s e_{1 k}(1),
\end{aligned}
$$


and we use them as we find it convenient. Now

$$
\begin{aligned}
& { }^{\alpha} e_{2 \sigma(k)}(*)={ }^{\alpha} s e_{k 1}(*) \\
& =s e_{1 k}(1) s e_{2 k}(\circledast) s e_{\sigma(k) k}(\circledast) s e_{k 1}(*) \\
& =s e_{1 k}(1) s e_{2 k}(\circledast)\left(s e_{\sigma(k) 1}(*) s e_{21}(*) s e_{k 1}(*)\right) \\
& =\operatorname{se}_{1 k}(1)\left(s e_{\sigma(k) 1}(*) s e_{21}(*)\right) \\
& =s e_{\sigma(k) k}(*) s e_{\sigma(k) 1}(*) s e_{2 k}(*) s e_{\sigma(k) k}(*) s e_{21}(*) \\
& =s e_{\sigma(k) k}(*) s e_{2 k}(*) s e_{21}(*) \text {, } \\
& { }^{\alpha} e_{k 2}(*)=\operatorname{se}_{2 k}(\circledast) s e_{\sigma(k) k}(\circledast) s e_{1 k}(1) s e_{k 2}(*) \\
& =\operatorname{se}_{2 k}(\circledast) s e_{\sigma(k) k}(\circledast)\left(s e_{12}(*) s e_{k 2}(*)\right) \\
& =\operatorname{se}_{2 k}(\circledast)\left(s e_{12}(*) s e_{\sigma(k) 2}(*) s e_{12}(*) s e_{k 2}(*)\right) \\
& =s e_{2 k}(\circledast)\left(s e_{12}(*) s e_{\sigma(k) 2}(*) s e_{k 2}(*)\right) \\
& =s e_{1 k}(*) s e_{\sigma(k) k}(*) s e_{12}(*) s e_{\sigma(k) k}(*) s e_{\sigma(k) 2}(*)\left({ }^{s e_{2 k}(\circledast)} s e_{k 2}(*)\right) \text {, } \\
& { }^{\alpha} s e_{k \sigma(k)}(*)=s e_{\sigma(k) k}(\circledast) s e_{2 k}(\circledast) s e_{1 k}(1) s e_{k \sigma(k)}(*) \\
& =s e_{\sigma(k) k}(\circledast) s e_{2 k}(\circledast)\left(s e_{k 2}(*) s e_{12}(*) s e_{k \sigma(k)}(*)\right) \\
& =X Y Z \text { (say), }
\end{aligned}
$$

where

$$
\begin{aligned}
& \left.X=s e_{\sigma(k) k}(\circledast) s e_{2 k}(\circledast)\right) s e_{k 2}(*) \\
& =s e_{2 k}(\circledast) s e_{\sigma(k) k}(\circledast) s e_{k 2}(*) \\
& =\operatorname{se}_{2 k}(\circledast)\left(\operatorname{se}_{\sigma(k) 2}(*) \operatorname{se}_{12}(*) \operatorname{se}_{k 2}(*)\right) \\
& =s e_{\sigma(k) k}(*) s e_{\sigma(k) 2}(*) s e_{1 k}(*) s e_{\sigma(k) k}(*) s e_{12}(*)\left({ }^{s e_{2 k}(\circledast)} s e_{k 2}(*)\right) \text {, } \\
& Y=s e_{\sigma(k) k}(\circledast) s e_{2 k}(\circledast) s e_{12}(*) \\
& =s e_{\sigma(k) k}(\circledast)\left(s e_{1 k}(*) s e_{\sigma(k) k}(*) s e_{12}(*)\right) \\
& =s e_{1 k}(*) s e_{\sigma(k) k}(*) s e_{12}(*) \text {, } \\
& \left.Z=s e_{\sigma(k) k}(\circledast) s e_{2 k}(\circledast)\right) s e_{k \sigma(k)}(*) \\
& =\operatorname{se}_{\sigma(k) k}(\circledast)\left(s e_{k 1}(*) s e_{21}(*) s e_{k \sigma(k)}(*)\right) \\
& =s e_{\sigma(k) 1}(*) s e_{21}(*) s e_{k 1}(*) s e_{21}(*)\left({ }^{s e_{\sigma(k) k}(\circledast)} s e_{k \sigma(k)}(*)\right) \text {. }
\end{aligned}
$$

Proof of Lemma 2.10, Let $f: \mathrm{E}^{1}(n, R, I) \longrightarrow \mathrm{E}^{1}(n, R / I, 0)$. Note that

$$
\operatorname{ker}(f) \subseteq \mathrm{E}^{1}(n, R, I) \cap \mathrm{G}(n, R, I) .
$$

Let $M=\prod g e_{j 1}\left(x_{j}\right) g e_{1 i}\left(a_{i}\right) \in \mathrm{E}^{1}(n, R, I) \cap \mathrm{G}(n, R, I)$. Note that $M \in \mathrm{G}(n, R, I)$ implies $\bar{M}=\prod g e_{j 1}(0) g e_{1 i}\left(\bar{a}_{i}\right)=\prod g e_{1 i}\left(\bar{a}_{i}\right)=I d$. Here bar means reduction modulo the ideal $I$. Therefore, $M \in \operatorname{ker}(f)$ and hence $\operatorname{ker}(f)=\mathrm{E}^{1}(n, R, I) \cap \mathrm{G}(n, R, I)$. 
Now we shall prove that $\operatorname{ker}(f)=\mathrm{E}(n, R, I)$. Let $E=\prod_{k=1}^{r} g e_{j_{k} 1}\left(x_{k}\right) g e_{1 i_{k}}\left(a_{k}\right) \in$ $\operatorname{ker}(f)$. Note that $E$ can be written as $g e_{j_{1} 1}\left(x_{1}\right) \prod_{k=2}^{r} \gamma_{k} g e_{j_{k} 1}\left(x_{k}\right) \gamma_{k}^{-1}$, where $\gamma_{l}$ is equal to $\prod_{k=1}^{l-1} g e_{1 i_{k}}\left(a_{k}\right) \in \mathrm{E}(n, R)$, and hence $\operatorname{ker}(f) \subseteq \mathrm{E}(n, R, I)$. The reverse inclusion follows from the fact that $\mathrm{E}(n, R, I) \subseteq \mathrm{E}^{1}(n, R, I)$.

Proof of Lemma 3.1 in the symplectic case when $n=4$ : Given that $s e_{i j}(X f(X))$ is in $\operatorname{ESp}_{2 n}^{1}(R[X], I[X])$. First assume $i=1$ and $f(X) \in R[X]$. We prove the result using induction on $r$, the number of generators of $\varepsilon$. Let $r=1$ and $\varepsilon=s e_{p q}(a)$. Note that when $p=1, a \in R$, and when $q=1, a \in I$. Also, note that we will use * to represent elements of the ideal $I$. Computation done in Case (1) to Case (11) in Lemma 3.1 will be the same here except Case (7). We work out the details of Case (7) now.

Case ( 7$)$ : Let $(p, q)=(j, 1)$ and $j \neq 2$. In this case

$$
\begin{aligned}
& s e_{j 1}(a) s e_{1 j}\left(Y^{4} X f\left(Y^{4} X\right)\right) s e_{j 1}(-a) \\
= & s e_{j 1}(a) s e_{\sigma(j) 2}\left(Y^{4} X f\left(Y^{4} X\right)\right) s e_{j 1}(-a) \\
= & s e_{j 1}(a)\left(\left[s e_{\sigma(j) j}\left(Y^{2}\right), s e_{j 2}\left(Y^{2} X f\left(Y^{4} X\right)\right)\right] s e_{12}\left((-1)^{j} Y^{6} X^{2} f^{2}\left(Y^{4} X\right)\right)\right) \\
= & {\left[s e_{\sigma(j) 1}\left(* Y^{2}\right) s e_{21}\left(* Y^{2}\right) s e_{\sigma(j) j}\left(Y^{2}\right), s e_{j \sigma(j)}\left(* Y^{2} X f\left(Y^{4} X\right)\right) s e_{j 2}\left(Y^{2} X f\left(Y^{4} X\right)\right)\right] } \\
& s e_{1 \sigma(j)}\left(* Y^{6} X^{2} f^{2}\left(Y^{4} X\right)\right) s e_{j \sigma(j)}\left(* Y^{6} X^{2} f^{2}\left(Y^{4} X\right)\right) s e_{12}\left((-1)^{j} Y^{6} X^{2} f^{2}\left(Y^{4} X\right)\right) \\
= & s e_{\sigma(j) 1}\left(* Y^{2}\right) s e_{21}\left(* Y^{2}\right)\left(s e_{\sigma(j) j}\left(Y^{2}\right) s e_{j \sigma(j)}\left(* Y^{2} X f\left(Y^{4} X\right)\right) s e_{j 2}\left(Y^{2} X f\left(Y^{4} X\right)\right)\right. \\
& \left.s e_{\sigma(j) j}\left(-Y^{2}\right)\right) s e_{21}\left(* Y^{2}\right) s e_{\sigma(j) 1}\left(* Y^{2}\right) s e_{j 2}\left(-Y^{2} X f\left(Y^{4} X\right)\right) \\
& s e_{j \sigma(j)}\left(* Y^{2} X f\left(Y^{4} X\right)\right) s e_{1 \sigma(j)}\left(* Y^{6} X^{2} f^{2}\left(Y^{4} X\right)\right) s e_{j \sigma(j)}\left(* Y^{6} X^{2} f^{2}\left(Y^{4} X\right)\right) \\
& s e_{12}\left((-1)^{j} Y^{6} X^{2} f^{2}\left(Y^{4} X\right)\right) \\
= & s e_{\sigma(j) 1}\left(* Y^{2}\right) s e_{21}\left(* Y^{2}\right)\left(s e_{\sigma(j) j}\left(Y^{2}\right)\left[s e_{j 1}(* Y), s e_{1 \sigma(j)}\left(* Y X f\left(Y^{4} X\right)\right)\right]\right. \\
& \left.s e_{\sigma(j) j}\left(Y^{2}\right) s e_{j 2}\left(Y^{2} X f\left(Y^{4} X\right)\right)\right) s e_{21}\left(* Y^{2}\right) s e_{\sigma(j) 1}\left(* Y^{2}\right) s e_{j 2}\left(-Y^{2} X f\left(Y^{4} X\right)\right) \\
& s e_{j \sigma(j)}\left(* Y^{2} X f\left(Y^{4} X\right)\right) s e_{1 \sigma(j)}\left(* Y^{6} X^{2} f^{2}\left(Y^{4} X\right)\right) s e_{j \sigma(j)}\left(* Y^{6} X^{2} f^{2}\left(Y^{4} X\right)\right) \\
& s e_{12}\left((-1)^{j} Y^{6} X^{2} f^{2}\left(Y^{4} X\right)\right) \\
= & s e_{\sigma(j) 1}\left(* Y^{2}\right) s e_{21}\left(* Y^{2}\right)\left[s e_{\sigma(j) 1}\left(* Y^{3}\right) s e_{21}\left(* Y^{4}\right) s e_{j 1}(* Y),\right. \\
& \left.s e_{\sigma(j) 2}\left(* Y^{3} X f\left(Y^{4} X\right)\right) s e_{12}\left(* Y^{4} X^{2} f^{2}\left(Y^{4} X\right)\right) s e_{1 \sigma(j)}\left(* Y X f\left(Y^{4} X\right)\right)\right] \\
& s e_{\sigma(j) 2}\left(Y^{4} X f\left(Y^{4} X\right)\right) s e_{12}\left(Y^{6} X^{2} f^{2}\left(Y^{4} X\right)\right) s e_{j 2}\left(Y^{2} X f\left(Y^{4} X\right)\right) s e_{21}\left(* Y^{2}\right) \\
& s e_{\sigma(j) 1}\left(* Y^{2}\right) s e_{j 2}\left(-Y^{2} X f\left(Y^{4} X\right)\right)\left[s e_{j 1}(* Y), s e_{1 \sigma(j)}\left(Y X f\left(Y^{4} X\right)\right)\right] \\
& s e_{1 \sigma(j)}\left(* Y^{6} X^{2} f^{2}\left(Y^{4} X\right)\right)\left[s e_{j 1}(* Y), s e_{1 \sigma(j)}\left(Y^{5} X^{2} f^{2}\left(Y^{4} X\right)\right)\right] \\
& s e_{12}\left((-1)^{j} Y^{6} X^{2} f^{2}\left(Y^{4} X\right)\right)
\end{aligned}
$$

Arguing as in the proof of Lemma 3.1 of $\S 3$, we can complete this proof.

Using equations (6) to (9) we can improve Theorem 5.6 of [7]. For this purpose we first establish the following lemma ([7, Lemma 5.2) for size 4: 
Lemma 7.10. Let $I$ be an ideal of $R$. Assume that $2 R=R$. Then $\operatorname{ESp}_{2 n}\left(R, I^{2}\right) \subseteq$ $\mathrm{ESp}_{2 n}(I)$, for $n \geq 2$.

Proof: Let $z^{*}=\sum a_{t} b_{t}$ with $a_{t}, b_{t} \in I$. Let $\beta=s e_{i j}\left(z^{*}\right) \in \operatorname{ESp}_{2 n}\left(I^{2}\right)$ and $\alpha=\operatorname{se}_{k l}(z) \in \operatorname{ESp}_{2 n}(R)$ for some $z \in R$. It suffices to show that $\alpha \beta \alpha^{-1} \in \operatorname{ESp}_{2 n}(I)$. If $(k, l) \neq(j, i)$ and $(k, l) \neq(\sigma(i), \sigma(j))$, then the matrix $\alpha \beta \alpha^{-1}$ splits into a product of elementary matrices from $\mathrm{ESp}_{2 n}(I)$. Now we consider the following two cases.

Case (1): Let $(k, l)=(j, i)$ or $(k, l)=(\sigma(i), \sigma(j))$, and $j \neq \sigma(i)$.

$$
\begin{aligned}
\alpha \beta \alpha^{-1}= & \prod s e_{j i}(z) s e_{i j}\left(a_{t} b_{t}\right) s e_{j i}(-z) \\
= & \prod s e_{j i}(z)\left(\left[s e_{i \sigma(i)}\left(a_{t}\right), s e_{\sigma(i) j}\left(b_{t}\right)\right] s e_{\sigma(j) j}\left((-1)^{i+j+1} a_{t} b_{t}^{2}\right)\right) \\
= & \prod\left[s e_{i \sigma(j)}\left(-a_{t} z\right) s e_{j \sigma(j)}\left((-1)^{i+j} a_{t} z^{2}\right) s e_{i \sigma(i)}\left(a_{t}\right), s e_{\sigma(i) i}\left(2 b_{t} z\right) s e_{\sigma(i) j}\left(b_{t}\right)\right] \\
& s e_{\sigma(j) i}\left((-1)^{i+j} a_{t} b_{t}^{2} z\right) s e_{\sigma(i) i}\left(-a_{t} b_{t}^{2} z^{2}\right) s e_{\sigma(j) j}\left((-1)^{i+j+1} a_{t} b_{t}^{2}\right) .
\end{aligned}
$$

Case (2): Let $(k, l)=(j, i)$ and $j=\sigma(i)$. Let us choose an integer $d \neq i, \sigma(i)$.

$$
\begin{aligned}
\alpha \beta \alpha^{-1} & =\prod s e_{\sigma(i) i}(z) s e_{i \sigma(i)}\left(a_{t} b_{t}\right) s e_{\sigma(i) i}(-z) \\
& =\prod e_{\sigma(i) i}(z)\left[s e_{i d}\left(a_{t}\right), s e_{d \sigma(i)}\left(b_{t} / 2\right)\right] \\
& =\prod\left[s e_{\sigma(i) d}\left(a_{t} z\right) s e_{\sigma(d) d}\left((-1)^{i+d+1} a_{t}^{2} z\right) s e_{i d}\left(a_{t}\right), s e_{d i}\left(b_{t} z / 2\right) s e_{d \sigma(i)}\left(b_{t} / 2\right)\right] .
\end{aligned}
$$

Now we have Lemma 5.1 to Lemma 5.4 and Theorem 5.5 of [7] for Elementary symplectic group of size 4 , which will prove the size 4 case of ([7], Theorem 5.6), viz.:

Theorem 7.11. (17, Theorem 5.6) Let $I$ be an ideal of $R$. Assume $2 R=R$. Then the natural map $\operatorname{Um}_{2 n}(R, I) / \mathrm{ESp}_{2 n}(R, I) \longrightarrow \mathrm{Um}_{2 n}(R, I) / \mathrm{E}_{2 n}(R, I)$ is bijective for $n \geq 2$.

\section{References}

[1] H. Apte, P. Chattopadhyay, R.A. Rao, A Local Global theorem for extended ideals, J. Ramanujan Math. Soc. 27, No. 1 (2012), 1-20.

[2] A. Bak, R. Basu, R.A. Rao, Local-Global principle for transvection groups, Proceedings of the American Mathematical Society 138 no. 4 (2010) 11911204

[3] H. Bass, $K$-theory and stable algebra, Inst. Publ. Math. IHES 22 (1964) $5-60$.

[4] H. Bass, Algebraic K-theory, W.A. Benjamin, Inc, 1968.

[5] H. Bass, Unitary algebraic $K$-theory, Lecture Notes in Mathematics 343 (1973) $57-265$ 
[6] R. Basu, R.A. Rao, Injective Stability for $K_{1}$ of Classical Modules, Journal of Algebra 323 no. 4 (2010) 867-877.

[7] P. Chattopadhyay, R.A. Rao, Elementary symplectic orbits and improved $K_{1}$-stability, Journal of $K$-Theory 7 (2011) 389-403.

[8] Hazrat, Roozbeh; Vavilov, Nikolai $K_{1}$ of Chevalley groups are nilpotent. J. Pure Appl. Algebra 179 (2003), no. 1-2, 99-116.

[9] Hazrat, R.; Petrov, V.; Vavilov, N. Relative subgroups in Chevalley groups. J. K-Theory 5 (2010), no. 3, 603-618.

[10] W. van der Kallen, A group structure on certain orbit sets of unimodular rows, Journal of Algebra 82 no. 2 (1983) 363-397.

[11] V.I. Kopeiko, The stabilization of symplectic groups over a polynomial ring, Math. USSR. Sbornik 34 (1978) 655-669.

[12] N. Kumar, M.P. Murthy, A. Roy, A cancellation Theorem for Projective Modules over Finitely Generated Rings, Algebraic Geometry and Commutative Algebra 1 (1988) 281-287.

[13] S. Mandal, Projective module and complete intersection, Lecture Notes in Mathematics 1672 (1997).

[14] D. Quillen, Projective modules over polynomial rings, Invent. Math. 36 (1976) $167-171$.

[15] R.A. Rao, An elementary transformation of a special unimodular vector to its top coefficient vector, Proceedings of the American Mathematical Society 93 no. 1 (1985) 21-24.

[16] A.A. Suslin, L.N. Vaserstein, Serre's problem on Projective Modules over Polynomial Rings and Algebraic K-theory, Math. USSR Izvestija 10 (1976) 937-1001.

[17] A.A. Suslin, On the Structure of the Special Linear Group over Polynomial Rings, Math. USSR. Izvestija 11 (1977) 221-238.

[18] A.A. Suslin, V.I. Kopeı̌ko, Quadratic Modules and Orthogonal Group over Polynomial Rings (Russian), Algebraic numbers and finite groups, Zap. Naučn. Sem. Lenin grad Otdel. Mat. Inst. Steklov. (LOMI ), 81 (123) No. 3 $1970328-351$.

[19] R.G. Swan, Serre's Problem, Queen's paper in pure and applied mathematics 42 Queen's University, Kingston, Ontario (1975) 1-60.

[20] L.N. Vaserstein, On the normal subgroups of $\mathrm{GL}_{n}$ over a ring, Algebraic $K$-theory, Evanston 1980 (Proc. Conf., Northwestern Univ., Evanston, Ill., 1980) 456-465; Lecture Notes in Math. 854, Springer, Berlin-New York, 1981. 